William Rodrigo Avendaño Castro*

Gerson Rueda Vera*** Luisa Stella Paz Montes****

Universidad Francisco de Paula Santander, Cúcuta, Colombia.

Recibido: 27 de mayo de 2015 Concepto de evaluación: 7 de diciembre de 2015 Aprobado: 9 de diciembre de 2015

Artículo de investigación (C) 2016 Universidad Católica de Colombia. Facultad de Ciencias Económicas y Administrativas. Todos los derechos reservados

* Doctor (c) en Ciencias Sociales y Humanas, Pontificia Universidad Javeriana, Bogotá, Colombia. Magíster en Comercio Internacional. Magíster en Administración. Especialista en Comercio Internacional. Especialista en Alta Gerencia. Economista en Comercio Exterior. Actualmente es profesor asociado de la Facultad de Ciencias Empresariales, Universidad Francisco de Paula Santander, Cúcuta, Colombia. Dirección de correspondencia: avenida 17E \# 0N-85, casa C5, Conjunto Villa Real. Código Postal: 540004088, Cúcuta,

Colombia. Correo electrónico: williamavendano@ufps.edu.co
Finanz. polit. econ., ISSN: 2248-6046, Vol. 8, No. 1, enero-junio, 2016, pp. 123-155

http://dx.doi.org/10.14718/revfinanzpolitecon.2016.8.1.7

\section{La gestión ambiental en las pymes del sector arcilla en Cúcuta y su área metropolitana*}

\section{RESUMEN}

Este artículo evalúa la gestión ambiental de las pequeñas y medianas empresas del sector arcilla presentes en el municipio de San José de Cúcuta y su área metropolitana. Para ello, se aplicó una encuesta a cuarenta empresas ubicadas en los municipios de Cúcuta, El Zulia, Villa del Rosario y Los Patios. La información recolectada se refirió a los instrumentos, las acciones y la gestión ambiental desarrollada por estas organizaciones. En su conjunto, los resultados se analizaron a través del componente de gestión ambiental del Modelo de Modernización para la Gestión de las Organizaciones. Se concluye que si bien las empresas presentan buenos indicadores en cada una de las variables, se deben exigir acciones preventivas y correctivas en aspectos como ruido, departamento de gestión ambiental, tratamiento de residuos sólidos, emisiones atmosféricas, ciclo de vida del producto, optimización de procesos y selección y uso de materias primas.

Palabras clave: gestión ambiental, pequeñas y medianas empresas, sector arcilla.

JEL: Q50, L70, O13

\section{Environmental management of SMEs in the clay sector in the city of Cúcuta, Colombia, and its metropolitan area}

\section{ABSTRACT}

This article evaluates environmental management by small and medium enterprises operating in the clay sector in the municipality of San José de Cúcuta, Colombia, and its metropolitan area. For the purpose, forty businesses in the municipalities of Cúcuta, El Zulia, Villa del Rosario and Los Patios were asked to respond to a questionnaire. Information was gathered on the

Este documento es producto de las actividades desarrolladas por el Grupo de Investigación en Ciencias Sociales y Humanas (GICSH) de la Universidad Francisco de Paula Santander. 
** Máster en Práctica Pedagógica. Máster en Gerencia de Empresas. Contador Público. Actualmente es docente de tiempo completo de la Facultad de Ciencias Empresariales, Universidad Francisco de Paula Santander, Cúcuta, Colombia. Dirección de correspondencia: calle 2, casa 2A-54, Urbanización Villa Camila. Código Postal: 540004088, Cúcuta, Colombia. Correo electrónico: gersonruedavera@ufps.edu.co

*** Estudiante de Doctorado en Educación. Magíster en Gerencia de Empresas. Especialista en Evaluación

Educativa con énfasis en Gestión de Proyectos y Administrador de Empresas. Actualmente se desempeña como docente de tiempo completo de la Facultad de Ciencias Empresariales, Universidad Francisco de Paula Santander, Cúcuta, Colombia. Dirección de correspondencia: calle 1 BN \# 16E-35, Parques Residenciales 3. Código postal: 540004088, Cúcuta, Colombia. Correo electrónico: luisastellapm@ufps.edu.co instruments and actions used by the firms in the general field of environmental management. The results were analyzed jointly using the environmental management component of the Model of Modernization for Organizational Management. The article concludes that while the indicators are positive for every variable, preventive and corrective measures should be put in place to ensure adequate noise control, the creation of dedicated environmental management departments in firms, appropriate solid waste treatment, the control of atmospheric emissions, attention to product life cycles, the optimization of processes, and the selection and use of raw materials.

Keywords: Environmental management, small and medium enterprises, clay sector.

\section{A gestão ambiental nas PMEs do setor de argila em Cúcuta e em sua área metropolitana}

\section{RESUMO}

Este artigo avalia a gestão ambiental das pequenas e médias empresas do setor de argila presentes no município de San José de Cúcuta (Colômbia) e em sua área metropolitana. Para isso, aplicou-se um questionário a quarenta empresas localizadas nos municípios de Cúcuta, El Zuila, Villa del Rosario e Los Patios. A informação coletada referiu-se aos instrumentos, às ações e à gestão ambiental desenvolvida por essas organizações. Em seu conjunto, os resultados foram analisados por meio do componente de gestão ambiental do Modelo de Modernização para a Gestão das Organizações. Conclui-se que, embora as empresas apresentem bons indicadores em cada uma das variáveis, devem-se exigir ações preventivas e corretivas em aspectos como barulho, departamento de gestão ambiental, tratamento de resíduos sólidos, emissões atmosféricas, ciclo de vida do produto, otimização de processos e seleção e uso de matérias-primas.

Palavras-chave: Gestão ambiental, pequenas e médias empresas, setor de argila. 


\section{INTRODUCCIÓN}

La actividad minera es una fuente de recursos que contribuyen al logro de la sostenibilidad de la población colombiana. Así sucede, en particular, en el departamento de Norte de Santander (Colombia), que se caracteriza por poseer un gran potencial minero, como el carbón, las arcillas, las calizas, los feldespatos y los fosfatos, materiales que actualmente se extraen y se transforman en la región.

La ubicación geográfica del departamento, en la que se destaca la posición fronteriza, la abundancia y la calidad de las arcillas, ha traído como consecuencia que en la región se haya instalado una gran cantidad de unidades productivas dedicadas a la fabricación de materiales cerámicos, principalmente aquellos destinados a la industria de la construcción. Esta concentración geográfica de industrias cerámicas hacen de este sector uno de los más estratégicos y promisorios para el departamento; las empresas, dedicadas al mercado local, regional, nacional y de exportación, en su conjunto son un importante generador de recursos que le imprime a la región una importante dinámica económica y social.

Sin embargo, son indiscutibles los problemas ambientales generados por el impacto de estas industrias. Según Montes-Guerra y Silva (2014), actualmente son mucho más evidentes las circunstancias y mucho más complejos los problemas que se relacionan con todas las fases del ciclo de vida de estos productos, desde la extracción de la materia prima, hasta la disposición y reutilización de los desechos del producto y de aquellos generados en su proceso de producción.

En efecto, en Norte de Santander se han presentado problemas de contaminación ambiental, deslizamientos y derrumbes en las carreteras, producto de los fenómenos de La Niña y El Niño; contaminación y agotamiento de sus cuencas hidrográficas; dos derramamientos de petróleo crudo en la quebrada Iscalá (abril de 2011) y en las corrientes del río Pamplonita (diciembre de 2011) que abastecen de agua potable en gran parte al municipio de Cúcuta; arrojo de desechos industriales a las cuencas hidrográficas, por la falta de previsión y de sistemas de control que mitiguen el impacto de la explotación, transporte y comercialización de recursos sobre el medioambiente.

En este entorno vital se interrelacionan elementos de diversa naturaleza: físicos, biológicos, económicos, culturales, sociales, entre otros (Gómez, 2002; Fernández y Leiva, 2003; Martínez, 2005), y en este panorama se ven envueltos los individuos, las comunidades y la sociedad en general. Para Gómez (2002), "la forma, el carácter, el comportamiento y la supervivencia" (p. 37) de uno se ven influenciados por los del otro, con referencia a la relación hombre-medioambiente. Por lo tanto, el futuro del hombre depende de las relaciones que establezca con la naturaleza y de su influencia positiva sobre ella.

El presente de estas relaciones está revestido de una evidente crisis, situación que ha conducido a que muchas personas, grupos e instituciones aboguen por reconciliar al hombre con el medio en el que habita. Para Brú (1997), la llamada crisis de la modernidad se trata de una "crisis de la verdad, o mejor, de la idea de la posibilidad de verdad" (p. 170), por cuanto el cambio de época transformó la verdad de la que era consciente el individuo, lo que modificó en él y en la sociedad su relación con el cosmos: "Ahora la recuperación, en la verdad del universo abolido, es imposible" (Sánchez, 1989, p. 24).

La inestabilidad de la que la humanidad es espectadora y, a la vez, protagonista ha permitido la construcción de nuevas formas de interpretación y de nuevos modelos culturales y sistemas de organización con la función de armonizar las relaciones entre el hombre y el medioambiente. La gestión ambiental es uno de esos campos con los que se pretende asumir esta problemática. Brú (1997) da una definición de gestión ambiental desde la perspectiva sociocultural:

[Es un] proceso de toma de decisiones acerca de cómo organizar la relación de la sociedad con la naturaleza y el medio ambiente. Los problemas, y con ellos, 
Wílliam Rodrigo Avendaño Castro • Gerson Rueda Vera • Luisa Stella Paz Montes

las soluciones no están en las cosas -en el medio y la naturaleza-, ni en nosotros -la sociedad - , sino en la interfase entre ambos (p. 19).

Moreno y Pol (1999) asumen la gestión ambiental desde un ámbito del diseño de la estrategia; la conciben como "aquella que incorpora los valores del desarrollo sostenible en las metas corporativas de la empresa o de la administración pública" (p. 13). Moreno y Pol (1999) señalan otros factores a la conceptualización de la gestión ambiental: a) la integración de políticas, programas y prácticas; b) la educación, enseñanza y motivación permanente de empleados y ciudadanos. La gestión ambiental está basada en el direccionamiento estratégico de la empresa, que según Conesa (1997) puede entenderse así:

El marco o el método de trabajo que sigue una organización con el objeto de conseguir [...] un determinado comportamiento de acuerdo con la metas que se hubiere fijado y como respuesta a unas normas, unos riesgos ambientales, y unas presiones tanto sociales, como financieras, económicas y competitivas, en permanente cambio (p. 61).

La gestión ambiental debe ser un compromiso ineludible de todo tipo de organizaciones. Según Conesa (1997), la gestión ambiental forma parte de la gestión empresarial, y son los directivos de las empresas quienes deben ocuparse de las actividades relacionadas con el medioambiente, a fin de contribuir a su conservación y a la creación de beneficios para los grupos de interés. Al reflexionar en torno a esta conceptualización, se encuentra la responsabilidad social empresarial (RSE) como una antesala de la gestión ambiental. En efecto, la RSE y la gestión ambiental, como lo señala McPhail (2000), es una "licencia social para que puedan operar las empresas en el mundo y en Colombia" (p. 48). Por su parte, Schmidheiny (2004) sostiene que las RSE se entiende como "el compromiso de las empresas de contribuir al desarrollo económico sostenible trabajando con los empleados, sus familias, la comunidad local y la sociedad en general para mejorar su calidad de vida".

Hasta hace poco, la actividad económica industrial no tenía en cuenta las repercusiones ambientales de su accionar en el medio físico y social que la rodeaba, situación que encuentra su génesis en la crisis de la modernidad y en los efectos desmedidos del modelo económico basado en el neoliberalismo. De este modo, la actividad empresarial ha estado desvinculada a la responsabilidad ambiental que deben tener las organizaciones.

La escasa conciencia en torno al tema de la gestión ambiental y la RSE de las organizaciones es causa de muchos de los fenómenos mencionados, situación que solo puede ser superada con el fortalecimiento de una cultura empresarial de protección del medioambiente. La poca conciencia para la implementación de sistemas de gestión ambiental en las empresas genera una baja relación costo-beneficio entre las erogaciones necesarias para prevenir o reparar los daños medioambientales y el valor del patrimonio público defendido o restaurado. Existe, por lo tanto, una necesidad de proponer alternativas ecológicas que conduzcan a una mayor productividad y competitividad en las empresas que integran el sector arcilla en Norte de Santander, pero con un enfoque que fomente una cultura de responsabilidad ambiental basada en la gestión ambiental.

Este artículo presenta los resultados de una investigación realizada en las pequeñas y medianas empresas (pyme) del sector de la arcilla del municipio de Cúcuta y su área metropolitana, a fin de evaluar la gestión ambiental allí realizada. Para este fin se aplicó un cuestionario con diversas variables a cuarenta empresas del municipio de Cúcuta, El Zulia, Villa del Rosario y Los Patios. La información recolectada fue analizada e incorporada dentro del Modelo de Modernización para la Gestión de las Organizaciones (MMGO) de la Universidad EAN de Colombia, a partir del componente de gestión ambiental para el diagnóstico, lo que permitió destacar las fortalezas y debilidades por cada una de las variables analizadas. Los resultados 
evidenciaron la necesidad de diseñar e implementar acciones, planes y programas tanto preventivos como correctivos en la gestión ambiental, sobre todo en aspectos como el ruido, el fortalecimiento de los departamentos de gestión ambiental, el tratamiento adecuado de los residuos sólidos y la mitigación de emisiones atmosféricas.

A continuación se hace un breve despliegue del marco teórico que sirvió de fundamento para el estudio, seguido de la metodología implementada. Luego se presentan los resultados, su análisis e interpretación. Por último se presentan las conclusiones y las recomendaciones, producto de la investigación.

\section{RESPONSABILIDAD SOCIAL EMPRESARIAL Y PEQUEÑAS $Y$ MEDIANAS EMPRESAS}

Al finalizar la década de 1970, como consecuencia de la creciente importancia de la empresa en el entorno socioeconómico, toma fuerza la doctrina relativa a la RSE, originada en las décadas de 1950 y 1960 en los Estados Unidos. Se manifiesta así un cambio de perspectiva social que produjo fuertes presiones sobre las empresas, por cuanto estas reclamaban "la consideración de las externalidades y costos sociales generados por su actividad $u$ objeto social, ante la constatación de la insuficiencia del beneficio socioeconómico de la empresa" (Momberg, 2008, p. 96). Este fue el punto de inicio de un profundo cambio en la conciencia social que impulsó la creación de exigencias concretas para las empresas y el auge de trabajos científicos en torno al fenómeno.

La palabra responsabilidad proviene del latín respondere, que significa 'responder' (Real Academia Española, 2007) a la exigencias y a las acciones. Por ello, el término solo puede ser referido a las acciones humanas. La responsabilidad es una carga, un compromiso o una obligación que los miembros de una sociedad, ya sea como individuos o como miembros de algún grupo, tienen tanto entre sí como para los colectivos. La RSE se fundamenta y se expresa en tres actitudes: respeto, justicia y solidaridad (Acosta, 2002).

La RSE, según Perdiguero y García (2005), tiene que ver con las "obligaciones legítimas hacia sus propietarios y accionistas, pero también con las responsabilidades innegables hacia el conjunto de individuos y grupos involucrados y/o afectados por sus actividades" (p. 19). La responsabilidad social concierne a la totalidad de las relaciones de las organizaciones con los grupos o individuos participantes (stakeholders) en cuatro niveles: a) relaciones con los trabajadores, b) calidad de la seguridad y utilidad social de los productos y servicios, c) creación y mantenimiento de los empleos y d) actitud responsable, de honestidad ante el impacto social, económico y ambiental de sus acciones. En estos niveles se formulan y desarrollan políticas, prácticas y programas que se instrumentan en toda la gama de operaciones corporativas y en los procesos de toma de decisiones.

Un grupo de interés, en su definición clásica, es "cualquier grupo o individuo que puede afectarse o es afectado por el logro de los objetivos de la organización" (Freeman, 1984, p. 46). Para Friedman y Miles (2006), la “organización en sí debe ser considerada como una agrupación de las partes interesadas y el propósito de la organización debe ser la gestión de sus intereses, necesidades y puntos de vista" (p. 11). Los grupos sobre los cuales las organizaciones (directivos y nivel superior) deben diseñar acciones específicas para la satisfacción de sus requerimientos pueden ser internos y externos. Dentro del primer grupo están los accionistas y los trabajadores, en tanto en el segundo se incluyen los clientes, los proveedores, los competidores, los agentes sociales, la comunidad local, las instituciones de administración pública, el Estado y el medioambiente.

La RSE tiene una relación íntima con el desarrollo de prácticas y acciones como parte de la filosofía de la organización, contexto en el que los grupos de interés son incluidos en los objetivos estratégicos. Estos son algunos aspectos compartidos entre los diversos autores, pues si bien se trata de un concepto abordado con gran amplitud, 
no tiene un sustrato conceptual homogéneo. Por ejemplo, Lindbaek (2003) explica que la RSE "es la responsabilidad que la corporación o la compañía tiene o asume frente a la sociedad en general" (p. 2). Encinas (2005) la observa como un instrumento de aceptación:

La RSE es una serie de conductas exigibles a las empresas por parte de esos interlocutores en función de la actividad que realiza, cuyo adecuado cumplimiento determinará que ésta sea aceptada o rechazada en sus diferentes aspectos (social económico, medioambiental) (p. 54).

Por su parte, Valor y De la Cuesta (2003) entienden la RSE de esta manera:

El conjunto de obligaciones y compromisos, legales y éticos, nacionales e internacionales, con los grupos de interés, que se derivan de los impactos que la actividad y operaciones de las organizaciones producen en el ámbito social, laboral, medioambiental y de los derechos humanos.

Por otro lado, Bigne et al. (2005) conceptualizan la RSE como un instrumento para "la creación de valor para los distintos grupos de interés que concurren en la actividad empresarial" (p. 16). La RSE tiene tres importantes razones para ser aplicada por las organizaciones: a) constituye un requisito en el mundo posmoderno, b) crea valor para la empresa y c) genera beneficios para los grupos de interés. Cegarra y Rodríguez (2004), con fundamento en el Libro Verde de la Comisión de las Comunidades Europeas, del 2002, y el Business Plan 2003-2005 de la Global Reporting Initiative, del 2003, indica que estos instrumentos "reconocen tanto la necesidad de aplicar prácticas sociales responsables como la obligación por parte de las empresas de informar a la sociedad de éstas" (p. 55).

De acuerdo con estos reportes, la RSE tiene una dimensión interna y otra externa. Las dos se encuentran conformadas por conocimientos: la dimensión interna contiene aquellos que existen dentro de la empresa para la gestión de la RSE, en tanto la dimensión externa agrupa los que existen entre la empresa y la sociedad (Hofer y Schendel, 1978).

La teoría relativa a la RSE no hace diferencias entre organizaciones por su tamaño u objeto social. Se trata de un conjunto de postulados que pueden ser aplicadas a cualquier empresa, ya que sus prácticas y sus beneficios pueden ser asumidos por estas. De acuerdo con Cabrera et al. (2002), la categoría "pyme es demasiado amplia y analíticamente engañosa porque esconde la heterogeneidad que caracteriza a este grupo de empresas" (p. 4); su variedad en todos los sentidos no permiten un análisis uniforme. En efecto, las pymes "se caracterizan por una mayor diversidad productiva" (Milesi y Moori, 2006, p. 55).

En Colombia, según la Ley 590 de 2000 y la Ley 905 de 2004, las pequeñas y medianas empresas son aquellas que cuentan con una planta de personal de entre 11 y 50 trabajadores, o activos totales entre 501 y menos de 5000 salarios mínimos mensuales legales vigentes. Las medianas empresas son aquellas que tienen una planta de personal de entre 51 y 200 trabajadores, o activos totales por valor de 5001 a 30.000 salarios mínimos mensuales legales vigentes.

Las pymes en Colombia representan un gran sector y tienen una especial atención por su contribución a la economía. De acuerdo con Aguirre y Córdoba (2008), este "grupo de empresas representa el $96 \%$ del total de empresas del país, proporciona el $65 \%$ del empleo y genera más del $35 \%$ del producto interno bruto (PIB)"' (p. 248). La importancia que revisten las pymes en Colombia y América Latina (Peres y Stumpo, 2002) obliga a que se desarrollen programas de RSE, no solo por la generación de valor, porque se trata de un requisito o por los beneficios a los grupos de interés, sino porque el éxito de la pyme está acompañado por la implementación de políticas de gestión y dirección sobre el talento humano, en función de crear una verdadera cultura organizacional 
(Rubio y Aragón, 2002). Otro factor por considerar para determinar la necesidad de planificar y desarrollar prácticas en torno a la RSE en el sector arcilla es que en este hay una tasa elevada de muertes, como lo expresa Crespi (2003).

\section{GESTIÓN AMBIENTAL}

Para Granero y Ferrando (2004), la definición de gestión ambiental no puede darse sin el abordaje del medioambiente como categoría teórica. Al igual que Gómez (2002), Granero y Ferrando (2004) consideran el medioambiente como mucho más que un conjunto de circunstancias físicas; lo definen como el "conjunto de circunstancias físicas que rodean a los seres vivos, o en una interpretación más amplia, como el conjunto de circunstancias físicas, culturales, sociales, económicas, etc., que rodean a las personas" (p. 11).

De allí es destacable el carácter social del concepto, por cuanto incluye las interrelaciones entre los diversos elementos. Por lo tanto, el medioambiente, considerado como un complejo conjunto de relaciones, permite considerar la gestión ambiental en un solo marco: el desarrollo sostenible, que es "la satisfacción de las necesidades del presente, sin comprometer la habilidad de las futuras generaciones para alcanzar sus propias metas" (Espinoza, 2007 , p. 17). En otras palabras, garantizar al hombre y al medio su existencia. Granero y Ferrando (2004) definen así la gestión ambiental:

Un conjunto de decisiones y acciones orientadas al logro del desarrollo sostenible, [cuyo objetivo es que] los niveles de calidad ambiental aumenten, [...] evitando y corrigiendo actividades que provocan degradación en el entorno, recuperando y restaurando los espacios degradados y potenciando los recursos ambientales y la capacidad de respuesta del medio ambiente (p. 12).

La gestión ambiental es una respuesta a los resultados de la Revolución Industrial, la cual ha
"Ilevado a un uso creciente del medio ambiente y los recursos naturales, lo cual ha contribuido a la toma de conciencia de que el entorno y los recursos naturales son realmente escasos" (Riera, 2005, p. 5). $Y$ esto se debe a las graves consecuencias sobre el medioambiente, el hábitat y las poblaciones, muchas de las cuales se han visto forzadas a desplazarse (Seoane, 2006).

Los recursos naturales son considerados patrimonio de la humanidad; por lo tanto, es un deber de las empresas, al hacer uso de sus medios, contribuir a su conservación. Para ello, es fundamental una acción conjunta por parte de las organizaciones: su compromiso activo en cada uno de sus niveles y trabajadores. La gestión ambiental, al igual que la RSE, es un asunto fundamental para gestar una cultura organizacional en este ámbito.

Así, una vez definida la gestión ambiental como acción y efecto de administrar el ambiente, se establece como un instrumento en el interior de la empresa que le permite definir y aplicar la normatividad ambiental y ecológica, al igual que delinear y dictar las políticas y estrategias ambientales y ecológicas propias.

\section{METODOLOGÍA}

Este estudio tiene un enfoque cuantitativo y es de tipo descriptivo. Al ser la minería el principal motor económico del país, se seleccionó para el presente estudio a las empresas que explotan arcilla, mineral no metálico, por ser el más representativo de Norte de Santander. Para el cumplimiento del objetivo, se identificó el número de pymes del sector, en los municipios de Cúcuta, El Zulia, Villa del Rosario y Los Patios. El número de empresas del sector arcilla en el municipio de Cúcuta y su área metropolitana es de 143 . Se presenta así un nivel de confianza del $95 \%$ y un margen de error de $5 \%$. La muestra seleccionada de forma no probabilística, de tipo accidental, estuvo conformada por 40 empresas distribuidas de la siguiente manera: 22 en Cúcuta (56\%), 8 en El Zulia (19\%), 6 en Villa del Rosario (15\%) y 4 en Los Patios (10\%). 
La recolección de la información se realizó a través de la aplicación de una encuesta de 15 preguntas (anexo 15) a los gerentes o jefes de producción de estas empresas ${ }^{1}$. En ella se incluyeron las siguientes variables: instrumentos de gestión ambiental preventivos y correctivos; programas de mitigación ambiental aplicados; perspectiva en torno al impacto ambiental de las empresas sobre el medioambiente y los recursos; diseño y desarrollo de proyectos ambientales; uso de la contabilidad ambiental; manejo de indicadores de gestión ambiental; programas de producción limpia; actualización de registros; uso de equipos de protección; uso de programas de prevención ambiental; programas dirigidos al bienestar de los grupos de interés y costos ambientales.

La información recolectada fue sistematizada e incluida de forma global en el componente de gestión ambiental del Modelo de Modernización para la Gestión de Organizaciones (MMGO) de la Universidad EAN. En este sentido, mencionan Pérez et al. (2011) que el $\mathrm{MMGO}^{2}$ ha sido implementado en más de 150 empresas, con el fin de hacer un análisis situacional basado en un enfoque sistémico y holístico. El MMGO incluye la valoración de diversos componentes como gestión del conocimiento, direccionamiento estratégico, cultura organizacional y gestión ambiental. Según Aguirre (2012), el componente de la gestión ambiental evalúa estas variables:

Sistemas de gestión ambiental, selección y uso de materias primas, optimización de procesos y programas de producción limpia, análisis del ciclo de vida del producto, residuos sólidos, aguas residuales, emisiones atmosféricas, olores, ruidos, publicidad externa visual, salud y seguridad industrial, registro y documentación,

$1 \quad$ Los resultados específicos de la aplicación de esta encuesta pueden ser solicitados a los autores del artículo a los correos electrónicos williamavendano@ufps.edu.co o wrac.phd@ gmail.com

2 El MMGO es una matriz que permite medir el estado actual de la gestión en las organizaciones. mejoramiento ambiental y departamento de gestión ambiental.

Cada una de estas variables contiene un conjunto de descriptores o indicadores que permite localizar la organización en uno de los cuatro estadios, donde el estadio 1 representa el de menor nivel de crecimiento, y el estadio 4, el de mayor nivel de crecimiento. A su vez, cada estadio se encuentra subdividido en tres posibles niveles: iniciando $(I)$, desarrollándose $(D)$ y madurando o en maduración $(M)$. La evaluación a partir del modelo MMGO permite ubicar a las empresas en estadios de acuerdo con la calificación obtenida, que va de 0 a 100. Una vez obtenidos los resultados por cada una de las variables que incorpora el MMGO al componente de gestión ambiental, se identifican los resultados globales, y a partir de ellos se pueden gestionar planes, programas y acciones de mejora de acuerdo con las prioridades establecidas. Las síntesis de los resultados, conforme a las variables descritas, se recopilan en las tablas 1 a 14, en tanto la información detallada de la aplicación de la matriz aparece en los anexos del artículo.

\section{RESULTADOS Y DISCUSIÓN}

En primer lugar, respecto a la variable gestión ambiental, hay acciones que identifican, evalúan y controlan, a través de la implementación de políticas ambientales, los impactos al entorno inmediato. Las empresas ejecutan programas que van orientados a su tratamiento y para los cuales existe un continuo seguimiento que incluye objetivos, metas y directrices. Las empresas han iniciado monitoreo, a través de personal, de la gestión ambiental, con el fin de identificar las diferentes tendencias que toma el análisis de impactos ambientales significativos (AIAS); sin embargo, no existe un sistema de acciones preventivas y correctivas que sean eficaces para su control. La calificación en esta variable es 71,43 (tabla 1).

Por otro lado, las empresas tienen en cuenta aspectos ambientales para la adquisición de materias primas y han empezado a considerar sus 
Tabla 1 .

Sintesis de los resultados: sistema de gestión ambiental

\begin{tabular}{|c|c|c|c|c|c|c|c|c|c|c|c|c|c|}
\hline \multirow{2}{*}{ Ítem } & \multirow{2}{*}{ Descriptores } & \multicolumn{3}{|c|}{ Estadio 1} & \multicolumn{3}{|c|}{ Estadio 2} & \multicolumn{3}{|c|}{ Estadio 3} & \multicolumn{3}{|c|}{ Estadio 4} \\
\hline & & I & D & $M$ & 1 & $\mathrm{D}$ & $\mathrm{M}$ & 1 & $\mathrm{D}$ & $\mathrm{M}$ & 1 & $\mathrm{D}$ & $\mathrm{M}$ \\
\hline \multirow{3}{*}{ Planeación } & $\begin{array}{c}\text { Evaluación de } \\
\text { aspectos e impactos } \\
\text { ambientales } \\
\text { significativos (AIAS) }\end{array}$ & & & & & & & & & & & 1 & \\
\hline & Política ambiental & & & & & & & & & & & 1 & \\
\hline & $\begin{array}{l}\text { Programas } \\
\text { ambientales }\end{array}$ & & & & & & & & & & & 1 & \\
\hline $\begin{array}{c}\text { Implementación } \\
\text { y control }\end{array}$ & $\begin{array}{c}\text { Monitoreo de los } \\
\text { impactos ambientales } \\
\text { significativos }\end{array}$ & & & & & & & 1 & & & & & \\
\hline \multirow[t]{2}{*}{ Verificación } & $\begin{array}{c}\text { Acciones preventivas y } \\
\text { correctivas }\end{array}$ & & & & 1 & & & & & & & & \\
\hline & Auditorias & & & & & & & & 1 & & & & \\
\hline $\begin{array}{l}\text { Revisión de } \\
\text { la dirección }\end{array}$ & Responsabilidades & & & & & & & & 1 & & & & \\
\hline \multicolumn{2}{|c|}{ Ponderación } & 0,00 & 0,00 & 0,00 & 0,33 & 0,00 & 0,00 & 0,58 & 1,33 & 0,00 & 0,00 & 2,75 & 0,00 \\
\hline \multicolumn{2}{|c|}{ Total posible } & \multicolumn{3}{|c|}{7,00} & & & & & & & & & \\
\hline \multicolumn{2}{|c|}{ Suma total } & \multicolumn{3}{|c|}{5,00} & & & & & & & & & \\
\hline \multicolumn{2}{|c|}{ Calificación } & \multicolumn{3}{|c|}{71,43} & & & & & & & & & \\
\hline
\end{tabular}

Fuente: elaboración de los autores.

características a la hora de darles uso; sin embargo, muestran falencias en cuanto a las cantidades que deben ser adquiridas, ya que estas se determinan según la experiencia, y aunque algunas son reusadas, estos procesos de recuperación no se documentan. La calificación es de 60,42 (tabla 2).

Respecto a la optimización de procesos y la producción más limpia, las empresas desconocen la cantidad de energía eléctrica que usan para la elaboración de sus productos, así como la contaminación causada por sus emisiones; no obstante, sí conocen la cantidad de agua utilizada en los procesos de manufacturación y emplean esta información para promover planes de ahorro del líquido. La calificación obtenida es de 50,00 (tabla 3).

Tabla 2.

Resultados en la selección y el uso de materias primas

\begin{tabular}{|c|c|c|c|c|c|c|c|c|c|c|c|c|}
\hline \multirow{2}{*}{ Descriptores } & \multicolumn{3}{|c|}{ Estadio 1 } & \multicolumn{3}{|c|}{ Estadio 2 } & \multicolumn{3}{c|}{ Estadio 3 } & \multicolumn{2}{c|}{ Estadio 4 } \\
\hline & I & D & M & I & D & M & I & D & M & I & D & M \\
\hline Selección & & & & & & & & & & & 1 & \\
\hline Caracterización & & & & & & & & 1 & & & & \\
\hline Uso & & & & & 1 & & & & & & & \\
\hline & & & & & 1 & & & & & & & \\
\hline Ponderación & 0,00 & 0,00 & 0,00 & 0,00 & 0,83 & 0,00 & 0,00 & 0,67 & 0,00 & 0,00 & 0,92 & 0,00 \\
\hline Total posible & \multicolumn{3}{|c|}{4,00} & & & & & & & & & \\
\hline Suma total & \multicolumn{3}{|c|}{2,42} & & & & & & & & & \\
\hline Calificación & \multicolumn{3}{|c|}{60,42} & & & & & & & & & \\
\hline
\end{tabular}

Fuente: elaboración de los autores. 
Tabla 3.

Resultados en la optimización de procesos y programas de producción más limpia

\begin{tabular}{|c|c|c|c|c|c|c|c|c|c|c|c|c|}
\hline \multirow{2}{*}{ Descriptores } & \multicolumn{3}{|c|}{ Estadio 1} & \multicolumn{3}{|c|}{ Estadio 2} & \multicolumn{3}{|c|}{ Estadio 3} & \multicolumn{3}{|c|}{ Estadio 4} \\
\hline & I & D & M & I & D & M & I & $\mathrm{D}$ & M & I & D & M \\
\hline Uso eficiente de la energía & & & 1 & & & & & & & & & \\
\hline Uso eficiente del agua & & & & & & & & & 1 & & & \\
\hline Ponderación & 0,00 & 0,00 & 0,25 & 0,00 & 0,00 & 0,00 & 0,00 & 0,00 & 0,75 & 0,00 & 0,00 & 0,00 \\
\hline Total posible & & 2,00 & & & & & & & & & & \\
\hline Suma total & & 1,00 & & & & & & & & & & \\
\hline Calificación & & 50,00 & & & & & & & & & & \\
\hline
\end{tabular}

Fuente: elaboración de los autores.

Por otro lado, en relación con la variable ciclo del producto, las empresas no llevan a cabo ningún tipo de análisis del uso de materias primas durante el proceso de manufacturación; no obstante, conocen los impactos ambientales que se generan tras la elaboración de productos, lo que ha contribuido al desarrollo de métodos de identificación con criterios que les permite la obtención de un sello ambiental. La calificación es 55,56 en esta variable (tabla 4).

Por otro lado, las empresas conocen y clasifican, según sus características, los residuos sólidos generados tras el proceso de producción, para su tratamiento según los parámetros establecidos por la ley; sin embargo, carecen de programas o planes ambientales que brinden una verdadera solución ecológica al tratamiento de estos residuos más allá de la normatividad. En cuanto a los restos posconsumo del producto, se han implementado programas (aún en fase beta) que les permiten a las empresas hacer un tratamiento apropiado del desecho que dejan los usuarios. La calificación es 50,00 en esta variable (tabla 5).

Tabla 4.

Análisis del ciclo de vida del producto o servicio

\begin{tabular}{|c|c|c|c|c|c|c|c|c|c|c|c|c|}
\hline \multirow{2}{*}{ Descriptores } & \multicolumn{3}{|c|}{ Estadio 1} & \multicolumn{3}{|c|}{ Estadio 2} & \multicolumn{3}{|c|}{ Estadio 3} & \multicolumn{3}{|c|}{ Estadio 4} \\
\hline & 1 & $\mathrm{D}$ & M & I & D & M & I & D & M & I & $\mathrm{D}$ & M \\
\hline $\begin{array}{c}\text { Evaluación de ciclo de vida asociado al uso } \\
\text { de materias primas }\end{array}$ & & & 1 & & & & & & & & & \\
\hline $\begin{array}{l}\text { Evaluación de ciclo de vida asociado al } \\
\text { análisis de impactos ambientales }\end{array}$ & & & & & & & & & & & 1 & \\
\hline Existencia de sellos ambientales & & & & & & 1 & & & & & & \\
\hline Ponderación & 0,00 & 0,00 & 0,25 & 0,00 & 0,00 & 0,50 & 0,00 & 0,00 & 0,00 & 0,00 & 0,92 & 0,00 \\
\hline Total posible & & 3,00 & & & & & & & & & & \\
\hline Suma total & & 1,67 & & & & & & & & & & \\
\hline Calificación & & 55,56 & & & & & & & & & & \\
\hline
\end{tabular}

Fuente: elaboración de los autores 
Tabla 5.

Resultados en residuos sólidos

\begin{tabular}{|c|c|c|c|c|c|c|c|c|c|c|c|c|}
\hline \multirow{2}{*}{ Descriptores } & \multicolumn{3}{|c|}{ Estadio 1} & \multicolumn{3}{|c|}{ Estadio 2} & \multicolumn{3}{|c|}{ Estadio 3} & \multicolumn{3}{|c|}{ Estadio 4} \\
\hline & I & D & $M$ & 1 & D & M & I & D & M & I & D & $M$ \\
\hline Caracterización & & & & & 1 & & & & & & & \\
\hline Valorización & & & & & 1 & & & & & & & \\
\hline Legislación & & & & & & & & & 1 & & & \\
\hline $\begin{array}{l}\text { Programas posconsumo (luminarias, RAES, } \\
\text { pilas y baterías, medicamentos, otros) }\end{array}$ & & & & & 1 & & & & & & & \\
\hline Ponderación & 0,00 & 0,00 & 0,00 & 0,00 & 1,25 & 0,00 & 0,00 & 0,00 & 0,75 & 0,00 & 0,00 & 0,00 \\
\hline Total posible & & 4,00 & & & & & & & & & & \\
\hline Suma total & & 2,00 & & & & & & & & & & \\
\hline Calificación & & 50,00 & & & & & & & & & & \\
\hline
\end{tabular}

Fuente: elaboración de los autores.

A su vez, las empresas poseen un sistema de tratamiento para las aguas residuales que hoy está en desarrollo y se ajusta a la normatividad legal; sin embargo, carecen de programas o planes ambientales que brinden una verdadera solución ecológica al tratamiento de estas aguas, más allá de las disposiciones establecidas por ley. La calificación de la variable tratamiento de aguas residuales es 70,83 (tabla 6).
Ahora bien, en la variable emisiones atmosféricas es posible evidenciar que las empresas poseen un sistema para el control y tratamiento de las emisiones contaminantes de la atmósfera; este proceso está aún en fase inicial y busca ajustarse a la normatividad legal. La calificación es 66,67 (tabla 7).

Tabla 6.

Resultados en aguas residuales

\begin{tabular}{|c|c|c|c|c|c|c|c|c|c|c|c|c|}
\hline \multirow{2}{*}{ Descriptores } & \multicolumn{3}{|c|}{ Estadio 1} & \multicolumn{3}{|c|}{ Estadio 2} & \multicolumn{3}{|c|}{ Estadio 3} & \multicolumn{3}{|c|}{ Estadio 4} \\
\hline & 1 & D & $M$ & I & $\mathrm{D}$ & M & I & $\mathrm{D}$ & M & 1 & D & M \\
\hline Caracterización & & & & & & & & 1 & & & & \\
\hline Legislación & & & & & & & & & 1 & & & \\
\hline Ponderación & 0,00 & 0,00 & 0,00 & 0,00 & 0,00 & 0,00 & 0,00 & 0,67 & 0,75 & 0,00 & 0,00 & 0,00 \\
\hline Total posible & & 2,00 & & & & & & & & & & \\
\hline Suma total & & 1,42 & & & & & & & & & & \\
\hline Calificación & & 70,83 & & & & & & & & & & \\
\hline
\end{tabular}

Fuente: elaboración de los autores. 
Tabla 7.

Resultados en emisiones atmosféricas

\begin{tabular}{|c|c|c|c|c|c|c|c|c|c|c|c|c|}
\hline \multirow{2}{*}{ Descriptores } & \multicolumn{3}{|c|}{ Estadio 1} & \multicolumn{3}{|c|}{ Estadio 2} & \multicolumn{3}{|c|}{ Estadio 3} & \multicolumn{3}{|c|}{ Estadio 4} \\
\hline & I & D & $\mathrm{M}$ & I & $\mathrm{D}$ & M & I & $\mathrm{D}$ & $\mathrm{M}$ & I & D & $\mathrm{M}$ \\
\hline Caracterización & & & & & & & 1 & & & & & \\
\hline Legislación & & & & & & & & & 1 & & & \\
\hline Ponderación & 0,00 & 0,00 & 0,00 & 0,00 & 0,00 & 0,00 & 0,58 & 0,00 & 0,75 & 0,00 & 0,00 & 0,00 \\
\hline Total posible & & 2,00 & & & & & & & & & & \\
\hline Suma total & & 1,33 & & & & & & & & & & \\
\hline Calificación & & 66,67 & & & & & & & & & & \\
\hline
\end{tabular}

Fuente: elaboración de los autores.

Además, las empresas poseen un sistema para el control y la supresión de olores. Sus indicadores permiten evaluar su efectividad y están ajustados a la normatividad legal, aunque su acción solo se limita a lo establecido por la ley. La calificación es 83,33 (tabla 8).
En lo atinente a la variable ruido, las empresas, aunque conocen la proveniencia de estos, no poseen programas para su control; solo se limitan a establecer las barreras básicas de disminución de sonidos establecidas por la ley. La calificación es 54,17 en esta variable (tabla 9).

Tabla 8.

Resultados en control de olores

\begin{tabular}{|c|c|c|c|c|c|c|c|c|c|c|c|c|}
\hline \multirow{2}{*}{ Descriptores } & \multicolumn{3}{|c|}{ Estadio 1} & \multicolumn{3}{|c|}{ Estadio 2} & \multicolumn{3}{|c|}{ Estadio 3} & \multicolumn{3}{|c|}{ Estadio 4} \\
\hline & I & D & M & I & D & $\mathrm{M}$ & 1 & $D$ & $\mathrm{M}$ & 1 & D & $\mathrm{M}$ \\
\hline Caracterización & & & & & & & & & & & 1 & \\
\hline Legislación & & & & & & & & & 1 & & & \\
\hline Ponderación & 0,00 & 0,00 & 0,00 & 0,00 & 0,00 & 0,00 & 0,00 & 0,00 & 0,75 & 0,00 & 0,92 & 0,00 \\
\hline Total posible & & 2,00 & & & & & & & & & & \\
\hline Suma total & & 1,67 & & & & & & & & & & \\
\hline Calificación & & 83,33 & & & & & & & & & & \\
\hline
\end{tabular}

Fuente: elaboración de los autores.

Tabla 9.

Resultados en control de ruido

\begin{tabular}{|c|c|c|c|c|c|c|c|c|c|c|c|c|}
\hline \multirow{2}{*}{ Descriptores } & \multicolumn{3}{|c|}{ Estadio 1} & \multicolumn{3}{|c|}{ Estadio 2} & \multicolumn{3}{|c|}{ Estadio 3} & \multicolumn{3}{|c|}{ Estadio 4} \\
\hline & 1 & D & M & I & D & $\mathrm{M}$ & 1 & D & $\mathrm{M}$ & 1 & D & M \\
\hline Caracterización & & & & & & 1 & & & & & & \\
\hline Legislación & & & & & & & 1 & & & & & \\
\hline Ponderación & 0,00 & 0,00 & 0,00 & 0,00 & 0,00 & 0,50 & 0,58 & 0,00 & 0,00 & 0,00 & 0,00 & 0,00 \\
\hline Total posible & & 2,00 & & & & & & & & & & \\
\hline Suma total & & 1,08 & & & & & & & & & & \\
\hline Calificación & & 54,17 & & & & & & & & & & \\
\hline
\end{tabular}

Fuente: elaboración de los autores. 
En la variable publicidad exterior visual, las empresas conocen y aplican la normatividad legal en cuanto al uso de publicidad excesiva y la contaminación visual que esta causa. La calificación es 75,00 en esta variable (tabla 10).

En la variable salud y seguridad industrial, las empresas se preocupan por la seguridad y el bienestar de sus empleados dentro y fuera de las instalaciones. Aparte de lo establecido por ley, en cuanto a salud, pensión, dotación y riesgos profesionales, las compañías procuran implementar programas de control, prevención y capacitación en riesgos y emergencias, para que sus empleados se desempeñen de manera óptima y eficaz en sus distintos puestos de trabajo. El seguimiento a los programas de seguridad y capacidad empresarial arrojan buenos resultados. La calificación es 97,92 (tabla 11).

Tabla 10 .

Resultados en control de publicidad exterior

\begin{tabular}{|c|c|c|c|c|c|c|c|c|c|c|c|c|}
\hline \multirow{2}{*}{ Descriptores } & \multicolumn{3}{|c|}{ Estadio 1} & \multicolumn{3}{|c|}{ Estadio 2} & \multicolumn{3}{|c|}{ Estadio 3} & \multicolumn{3}{|c|}{ Estadio 4} \\
\hline & I & D & $\mathrm{M}$ & I & D & $\mathrm{M}$ & I & D & M & I & D & M \\
\hline Legislación & & & & & & & & & 1 & & & \\
\hline Ponderación & 0,00 & 0,00 & 0,00 & 0,00 & 0,00 & 0,00 & 0,00 & 0,00 & 0,75 & 0,00 & 0,00 & 0,00 \\
\hline Total posible & & 1,00 & & & & & & & & & & \\
\hline Suma total & & 0,75 & & & & & & & & & & \\
\hline Calificación & & 75,00 & & & & & & & & & & \\
\hline
\end{tabular}

Fuente: elaboración de los autores.

Tabla 11

Resultados en salud y seguridad industrial

\begin{tabular}{|c|c|c|c|c|c|c|c|c|c|c|c|c|}
\hline \multirow{2}{*}{ Descriptores } & \multicolumn{3}{|c|}{ Estadio 1} & \multicolumn{3}{|c|}{ Estadio 2} & \multicolumn{3}{|c|}{ Estadio 3} & \multicolumn{3}{|c|}{ Estadio 4} \\
\hline & 1 & $\mathrm{D}$ & $\mathrm{M}$ & 1 & $\mathrm{D}$ & M & I & $\mathrm{D}$ & M & I & D & M \\
\hline Afiliación & & & & & & & & & & & & 1 \\
\hline Dotación & & & & & & & & & & & & 1 \\
\hline Planes de emergencia y contingencia & & & & & & & & & & & 1 & \\
\hline Capacitación & & & & & & & & & & & & 1 \\
\hline Ponderación & 0,00 & 0,00 & 0,00 & 0,00 & 0,00 & 0,00 & 0,00 & 0,00 & 0,00 & 0,00 & 0,92 & 3,00 \\
\hline Total posible & & 4,00 & & & & & & & & & & \\
\hline Suma total & & 3,92 & & & & & & & & & & \\
\hline Calificación & & 97,92 & & & & & & & & & & \\
\hline
\end{tabular}

Fuente: elaboración de los autores.

Adicionalmente, las empresas llevan registros sistematizados de manera ordenada desde hace más de doce años, lo que les permite hacer una planeación oportuna en cuanto a planes de mejoramiento en sus distintas áreas; no obstante, en algunos casos esta información no es analizada, y en el caso específico de la reutilización de materia prima, esta no está documentada. La calificación de la variable registros y documentación es de 91,67 (tabla 12). 
Por otro lado, las empresas, aunque son conscientes de los diferentes impactos ambientales causados por sus actividades de producción y poseen objetivos para disminuir la contaminación causada, hoy carecen de un plan de mejoramiento ambiental. Aun así, se trabaja en la elaboración de un plan guiado por las distintas acciones implementadas, como medida de control para salvaguardar el medioambiente afectado por la manufacturación de sus productos. La calificación de la variable mejoramiento ambiental es 70,83 (tabla 13).
Por último, se evidencia que las empresas han instituido departamentos encargados de velar por la salvaguarda del medioambiente. A medida que pasa el tiempo, estos se fortalecen; de hecho, se encuentran en una etapa de desarrollo hacia su consolidación como mediadores, protectores y controladores de los aspectos industriales que afecten de forma negativa el entorno ecológico de la empresa. La calificación de la variable Departamento de Gestión Ambiental es 66,67 (tabla 14).

Tabla 12.

Resultados en registros y documentación

\begin{tabular}{|c|c|c|c|c|c|c|c|c|c|c|c|c|}
\hline \multirow{2}{*}{ Descriptores } & \multicolumn{3}{|c|}{ Estadio 1} & \multicolumn{3}{|c|}{ Estadio 2} & \multicolumn{3}{|c|}{ Estadio 3} & \multicolumn{3}{|c|}{ Estadio 4} \\
\hline & 1 & $\mathrm{D}$ & $\mathrm{M}$ & $\mathrm{I}$ & $\mathrm{D}$ & $\mathrm{M}$ & 1 & $\mathrm{D}$ & $\mathrm{M}$ & 1 & D & $M$ \\
\hline Sistema de documentación & & & & & & & & & & & 1 & \\
\hline Ponderación & 0,00 & 0,00 & 0,00 & 0,00 & 0,00 & 0,00 & 0,00 & 0,00 & 0,00 & 0,00 & 0,92 & 0,00 \\
\hline Total posible & \multicolumn{3}{|c|}{1,00} & & & & & & & & & \\
\hline Suma total & \multicolumn{3}{|c|}{0,92} & & & & & & & & & \\
\hline Calificación & \multicolumn{3}{|c|}{91,67} & & & & & & & & & \\
\hline
\end{tabular}

Fuente: elaboración de los autores.

Tabla 13.

Resultados en planes de mejoramiento ambiental

\begin{tabular}{|c|c|c|c|c|c|c|c|c|c|c|c|c|}
\hline \multirow{2}{*}{ Descriptores } & \multicolumn{3}{|c|}{ Estadio 1} & \multicolumn{3}{|c|}{ Estadio 2} & \multicolumn{3}{|c|}{ Estadio 3} & \multicolumn{3}{|c|}{ Estadio 4} \\
\hline & 1 & $\mathrm{D}$ & $\mathrm{M}$ & $\mathrm{I}$ & $\mathrm{D}$ & $\mathrm{M}$ & 1 & $\mathrm{D}$ & $\mathrm{M}$ & $\mathrm{I}$ & $\mathrm{D}$ & $\mathrm{M}$ \\
\hline Plan de Manejo Ambiental (PMA) & & & & & & 1 & & & & & & \\
\hline Informes de Cumplimiento Ambiental (ICA) & & & & & & & & & & & 1 & \\
\hline Ponderación & 0,00 & 0,00 & 0,00 & 0,00 & 0,00 & 0,50 & 0,00 & 0,00 & 0,00 & 0,00 & 0,92 & 0,00 \\
\hline Total posible & & 2,00 & & & & & & & & & & \\
\hline Suma total & & 1,42 & & & & & & & & & & \\
\hline Calificación & & 70,83 & & & & & & & & & & \\
\hline
\end{tabular}

Fuente: elaboración de los autores.

Tabla 14.

Resultados en existencia y funcionamiento de Departamento de Gestión Ambiental

\begin{tabular}{|c|c|c|c|c|c|c|c|c|c|c|c|c|}
\hline \multirow{2}{*}{ Descriptores } & \multicolumn{3}{|c|}{ Estadio 1} & \multicolumn{3}{|c|}{ Estadio 2} & \multicolumn{3}{|c|}{ Estadio 3} & \multicolumn{3}{|c|}{ Estadio 4} \\
\hline & 1 & D & $\mathrm{M}$ & I & $\mathrm{D}$ & $\mathrm{M}$ & I & $\mathrm{D}$ & $\mathrm{M}$ & 1 & $\mathrm{D}$ & $\mathrm{M}$ \\
\hline Departamento de Gestión Ambiental & & & & & & & & 1 & & & & \\
\hline Ponderación & 0,00 & 0,00 & 0,00 & 0,00 & 0,00 & 0,00 & 0,00 & 0,67 & 0,00 & 0,00 & 0,00 & 0,00 \\
\hline Total posible & & 1,00 & & & & & & & & & & \\
\hline Suma total & & 0,67 & & & & & & & & & & \\
\hline Calificación & & 66,67 & & & & & & & & & & \\
\hline
\end{tabular}

Fuente: elaboración de los autores. 
Gráfica 1.

Resumen de resultados de la aplicación MMGO en las diferentes variables de la dimensión gestión ambiental

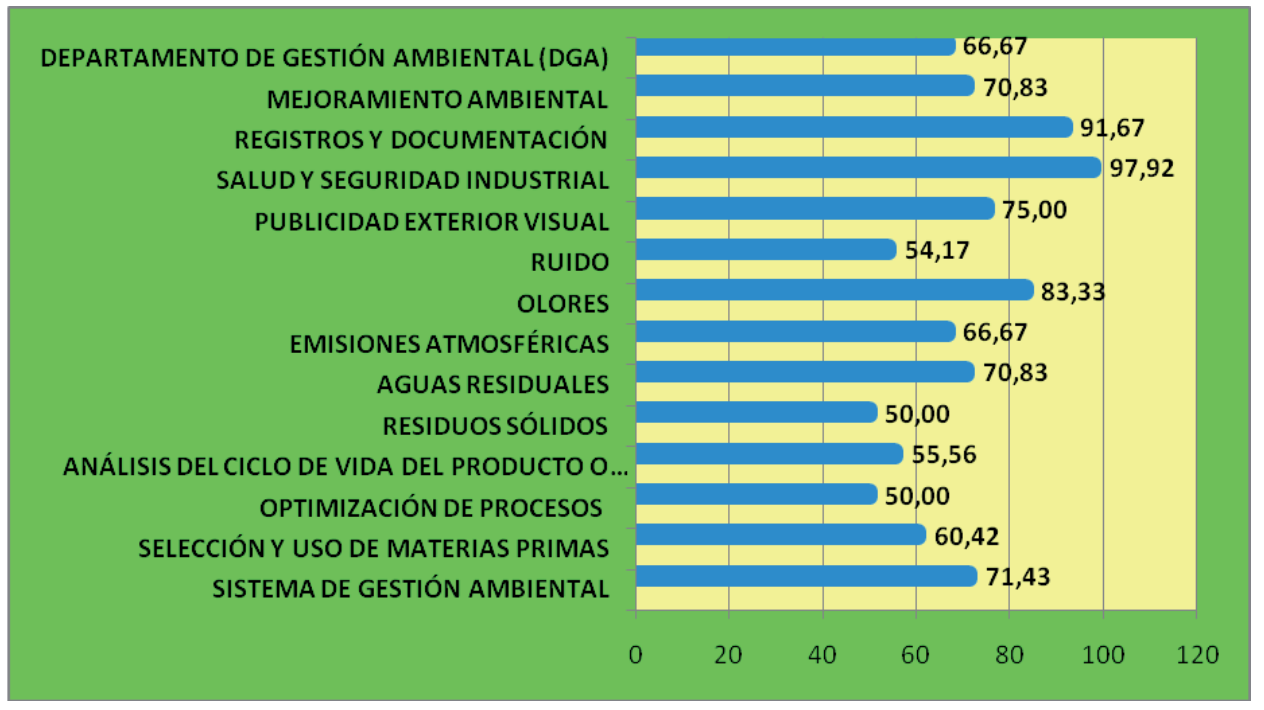

Fuente: elaboración de los autores a partir de Pérez et al. (2011).

En la gráfica anterior se resumen los resultados obtenidos en las 14 variables en estudio.

Las fortalezas están determinadas por los procesos de gestión ambiental en los que las empresas del sector arcilla se encuentran en estadio 4:

- En cuanto a salud y seguridad industrial, las empresas no se limitan a lo legal, sino que van un paso adelante para brindar un bienestar completo a sus trabajadores. Por ello, tienen en cuenta aspectos que van desde una dotación adecuada y pertinente para el ejercicio de sus funciones, hasta capacitaciones en planes de emergencia y contingencia en caso de presentarse riesgos.

- Para la elaboración de planes de mejoramiento, gestión ambiental y aspectos relacionados, se utiliza documentación de más de un año y se tienen en cuenta las experiencias pasadas, para superar errores y encontrar mejoras en los resultados. Toda esta documentación está organizada de forma debida tanto en medios físicos como magnéticos, para su fácil ubicación y uso.
Las oportunidades de mejoramiento están dadas por los procesos de gestión ambiental, en los cuales las empresas del sector arcilla se encuentran en estadio 1 y 2 , de acuerdo con el MMGO:

- El sistema de gestión ambiental de las empresas del sector arcilla del de San José de Cúcuta y su zona metropolitana se encuentra en buen nivel en cuanto a la identificación de los AIAS y los métodos de tratamiento para su control. Existe también una gran posibilidad de que el personal encargado del sistema de gestión ambiental fortalezca las acciones preventivas asociadas a los AIAS, lo que permite documentar las operaciones para que esta información se convierta en la base de futuras gestiones.

- En cuanto al tratamiento de aguas residuales, existe una gran oportunidad de optimizar las acciones de las empresas en esta materia, para crear planes innovadores que coadyuven a un tratamiento más eficaz.

- La buena puntuación obtenida en la matriz para el tratamiento de olores 
establece una pauta para trascender más allá de los parámetros establecidos por la ley en cuanto a este aspecto. Las empresas, con base en esta fortaleza, pueden generar un programa ambiental que transcienda el bienestar descrito en las normas en cuanto al control y la disminución de olores, en aras de crear un ecosistema de mayor salubridad.

- Con respecto a la publicidad exterior visual, no basta con cumplir con los parámetros establecidos por la ley; la mejor forma de potenciar este aspecto es establecer nuevas formas de hacer publicidad a través de las nuevas tecnologías, para que así los campos abiertos no estén invadidos de imágenes excesivas que recarguen o destruyan los paisajes.

- Si bien se tienen en cuenta los informes de las acciones llevadas a cabo para disminuir el impacto ambiental de las empresas sobre los ecosistemas, se hace perentorio la creación e implementación de un Plan de Manejo Ambiental (PMA) que lleve a las organizaciones a un constante mejoramiento.

- Las empresas comprenden los diferentes impactos ambientales que sus procesos generan en los ecosistemas; de allí la constitución de departamentos de gestión ambiental, encargados de promover acciones que disminuyan los impactos causados y promuevan la recuperación del medio. Adicionalmente, estos necesitan llevar un recuento organizado de sus labores y las formas como las gestionan en pro del ambiente, para sustentar ante entes encargados y autoridades competentes los planes y programas que se llevan a cabo en cuanto a identificación, evaluación, tratamiento y control de los impactos ambientales generados.

\section{CONCLUSIONES Y RECOMENDACIONES}

La gestión ambiental se ha convertido en una licencia para operar, aumentar la competitividad y mejorar las condiciones de vida de los grupos de interés relacionados de forma directa e indirecta con las organizaciones. Si bien en las empresas analizadas del sector arcilla del municipio de Cúcuta y su área metropolitana se gestionan acciones para identificar, evaluar y controlar aspectos e impactos ambientales significativos, se observa que en general estas acciones tienen su origen en criterios legales.

Por otro lado, las empresas no logran responder y actuar con todos los grupos humanos con los que están ligadas, así como con el medioambiente. Tampoco alcanzan un verdadero compromiso con el bien social si no cuentan con una filosofía social, un convencimiento de la dirección y un compromiso corporativo. La mayoría de las prácticas observadas en pro de la gestión ambiental y la responsabilidad social empresarial responden más a intereses legales.

Se observa así que lo que implica la gestión ambiental respecto a la incorporación de los valores del desarrollo sostenible dentro de los objetivos empresariales no responde a una convicción ni ha sido incorporado como parte de la identidad empresarial. Aunque las empresas analizadas demuestran fortalezas en el ámbito de la gestión ambiental, es necesario trabajar con el desarrollo de las oportunidades y la creación de nuevas estrategias para incluir acciones diversas y efectivas que contribuyan en el desarrollo sostenible, desde la cultura organizacional y la responsabilidad social empresarial.

Ahora bien, conviene señalar algunas recomendaciones fundamentales, resultado del estudio:

- Dentro de los datos analizados se destaca que la responsabilidad social empresarial implica el cuidado del medioambiente, para lo cual ha de evitarse la 
contaminación y el mal uso de recursos. Es necesario implementar programas que permitan conocer la cantidad de energía eléctrica usada durante el proceso de producción, además de las emisiones de carbono producidas en esta actividad, para así elaborar planes que gestionen el uso considerado de la energía o supongan el uso de energías alternas para controlar las emisiones contaminantes.

- Desde el mismo criterio, se deberá trabajar para que la dirección de gestión ambiental de las empresas perfeccione el tratamiento de los residuos sólidos y su correcta clasificación, según su grado potencial de contaminación. El objetivo será crear programas que ejecuten distintas maneras de eliminación de residuos, de acuerdo con los peligros que estos generan al medioambiente, o bien, gestionar su reutilización, en caso de que sea posible. Por otra parte, se deben crear campañas posconsumo que culturicen a los usuarios finales de los productos: que al desecharlos, la empresa puede encargarse de ellos, de forma gratuita, lo que contribuye a que las personas adquieran una conciencia de preservación del medioambiente.

- El control de ruido es otro de los aspectos en el que las empresas del sector arcilla de Cúcuta y su área metropolitana obtuvieron una baja puntuación; por esta razón es importante que, una vez identificados los orígenes de los fuertes ruidos, se creen barreras que los aparten, los detengan o los aíslen, para disminuir los índices de contaminación auditiva causados. Todo lo anterior ha de estar regulado por un estricto seguimiento de control de calidad, que hacia futuro sirva como base para la elaboración de planes de mejoramiento que permitan fortalecer las acciones sobre las fuentes de sonidos estruendosos.

- Es primordial no solo determinar y documentar las cantidades necesarias de materias primas utilizadas durante el proceso de producción, sino también asegurar que los datos sean analizados para planificar la producción, además de implementar programas concretos que permitan el reúso de materias primas y el establecimiento de criterios ambientales para la adquisición de las estas.

- En relación con esto último, se debe comenzar a realizar un balance de las materias primas utilizadas en el proceso de producción, para desarrollar indicadores operacionales con respecto al consumo de energía, agua o cantidad de productos elaborados con respecto a la cantidad de materiales. Además, como ya están identificados los impactos ambientales causados por las empresas, debe trabajarse en la elaboración e implementación de planes que constituyan un camino seguro hacia la obtención de un sello que certifique la buena gestión del medioambiente realizada por la organización.

- En lo que respecta a la responsabilidad social, se deberá ir más allá del cumplimiento estricto de las normas, para generar una verdadera contribución. Se observa un nivel de compromiso por parte de empresas para desarrollar diversos programas que favorezcan el bienestar social, el cual deberá ser profundizado y materializado, para que se convierta en parte de la identidad empresarial. 


\section{REFERENCIAS}

1. Acosta, R. (2002). Inserción del eje transversal responsabilidad social en los planes de estudio de la Universidad Metropolitana. Revista Anales, 2(2), 51-71.

2. Aguirre, L. (2012). Propuesta modelo de intervención e innovación para la gestión de organizaciones (MMGO) componente de gestión ambiental (tesis de grado). Bogotá: Universidad EAN.

3. Aguirre, S. y Córdoba, N. (2008). Diagnóstico de la madurez de los procesos en empresas medianas colombianas. Ingeniería y Universidad, 12 (2), 245-267.

4. Bigné, E. et al. (2005). Percepción de la responsabilidad social corporativa: un análisis cross-cultural. Universia Business Review, 5, 14-27.

5. Brú, J. (1997). Medio ambiente: poder y espectáculo. Barcelona: Icaria.

6. Cabrera, Á. et al. (2002). Las pyme: quiénes son, cómo son y qué hacer con ellas. Recuperado de https://www. researchgate.net/publication/42532811_Las_PyME_Quienes_Son_Como_Son_y_Que_Hacer_Con_Ellas

7. Cegarra, J. G. y Rodríguez, J. M. (2004). Prácticas de gestión social y componentes de la responsabilidad social corporativa. Cuaderno de Administración, 17(28), 53-70.

8. Conesa, V. (1997). Instrumentos de la gestión ambiental en la empresa. Madrid: Mundi-Prensa.

9. Congreso Nacional de la República (2000). Ley 590, por la cual se dictan disposiciones para promover el desarrollo de las micro, pequeñas y medianas empresas. Diario Oficial 44.078.

10. Congreso Nacional de la República (2004). Ley 905 de 2004, por la cual se modifica la Ley 590 de 2000 sobre promoción del desarrollo de la micro, pequeña y mediana empresa colombiana y se dictan otras disposiciones. Diario Oficial 45.228.

11. Crespi, G. (2003). Pyme en Chile: nace, crece y muere. Análisis de su desarrollo en los últimos siete años. Santiago de Chile: FUNDES.

12. Encinas, B. (2005). Marco conceptual de la responsabilidad social de las empresas. La dimensión social de la empresa. X Jornadas de Investigadores en Economía Social y Cooperativa: Emprendedores, Economía Social, Democracia Económica. Andalucía: Universidad de Jaén.

13. Espinoza, G. (2007). Gestión y fundamentos de evaluación de impacto ambiental. Santiago de Chile: Banco Interamericano de Desarrollo (BID) y Centro de Estudios para el Desarrollo (CED.

14. Fernández, R. y Leiva, M. (2003). Ecología para la agricultura. Madrid: Mundi-Prensa.

15. Freeman, E. (1984). Strategic management: a stakeholder approach. Boston: Pitman.

16. Friedman, A. y Miles, S. (2006). Stakeholders. Theory and practice. Nueva York: Oxford University Press.

17. Global Reporting Initiative (2003). Business Plan 2003-2005. Ámsterdam: Autor.

18. Gómez, D. (2002). Evaluación de impacto ambiental. Madrid: Mundi-Prensa.

19. Granero, J. y Ferrando, M. (2004). Cómo implantar un sistema de gestión ambiental según la norma ISO 14001:2004. Madrid: Fundación Confemetal.

20. Hofer, C. y Schendel, D. (1978). Strategy formulation: analytical concepts. Los Ángeles: West Publishing Company.

21. Lindbaek, J. (2003). La ética y la responsabilidad social empresarial. Seminario Internacional Capital Social, Ética y Desarrollo: Los Desafíos de la Gobernabilidad Democrática. Caracas: Iniciativa Interamericana de Capital Social, Ética y Desarrollo. 
22. Martínez, V. (2005). ESO 4. Libro de educación física. Barcelona: Paidotribo.

23. McPhail, K. (2000). How oil, gas and mining projects can contribute to development. Finance and Development, 37(4), 46-50.

24. Milesi, D. y Moori, V. (2006). Éxito exportador en la pymes colombianas. En V. Moori (comp.), Perfil de la PYME exportadora exitosa: el caso colombiano (pp. 55-69). Bogotá, FUNDES.

25. Momberg, M. (2008). Responsabilidad social empresarial (RSE) como ventaja competitiva (p. 95-111). En L. Marchant (Ed.), Actualizaciones para el management y el desarrollo organizacional (pp. 95-11). Valparaíso: Universidad Viña del Mar.

26. Montes-Guerra, M. y Silva, H. (2014). Implementación de procesos sostenibles vinculando industrias regionales: Reciclaje de residuos siderúrgicos como proyecto de cambio de la mampostería en BoyacáColombia. Revista EAN, 77.

27. Moreno, E. y Pol, E. (1999). Nociones psicosociales para la intervención y la gestión ambiental. Barcelona: Universidad de Barcelona.

28. Perdiguero, T. y García, A. (Eds.) (2005). La responsabilidad social de las empresas y los nuevos desafíos de la gestión empresarial. Barcelona: Universidad de Valencia.

29. Peres, W. y Stumpo, G. (2002). Las pequeñas y medianas empresas industriales en América Latina y el Caribe. Ciudad de México: Siglo XXI.

30. Pérez, R. et al. (2011). MMGO: Modelo de Modernización para la Gestión de Organizaciones. Bogotá: EAN.

31. Real Academia Española (2007). Diccionario de la lengua española. Barcelona: Espasa.

32. Riera, P. (2005). Manual de economía ambiental y de los recursos naturales. Madrid: Paraninfo.

33. Rubio, A. y Aragón, A. (2002). Factores explicativos del éxito competitivo. Un estudio empírico en la pyme. Cuadernos de Gestión, 2(1), 49-63.

34. Sánchez, D. (1989). En torno al Superhombre. Nietszche y la crisis de la modernidad. Barcelona: Anthropos.

35. Schmidheiny, S. (2004). La visión del empresario para el desarrollo sostenible a través de la responsabilidad social. Recuperado de http://anep.org.sv/ENADE2004/docenade04.pdfi

36. Seoane, J. (2006). Movimientos sociales y recursos naturales en América Latina: resistencias al neoliberalismo, configuración de alternativas. Sociedade e Estado, 21 (1), 85-107.

37. Valor, C. y De la Cuesta, M. (2003). Responsabilidad social de la empresa. Concepto, medición y desarrollo en España. Recuperado de http://www.revistasice.info/cachepdf/BICE_2755_07-19_843B2AFA16833 BD45F65BF48332D2587.pdf 


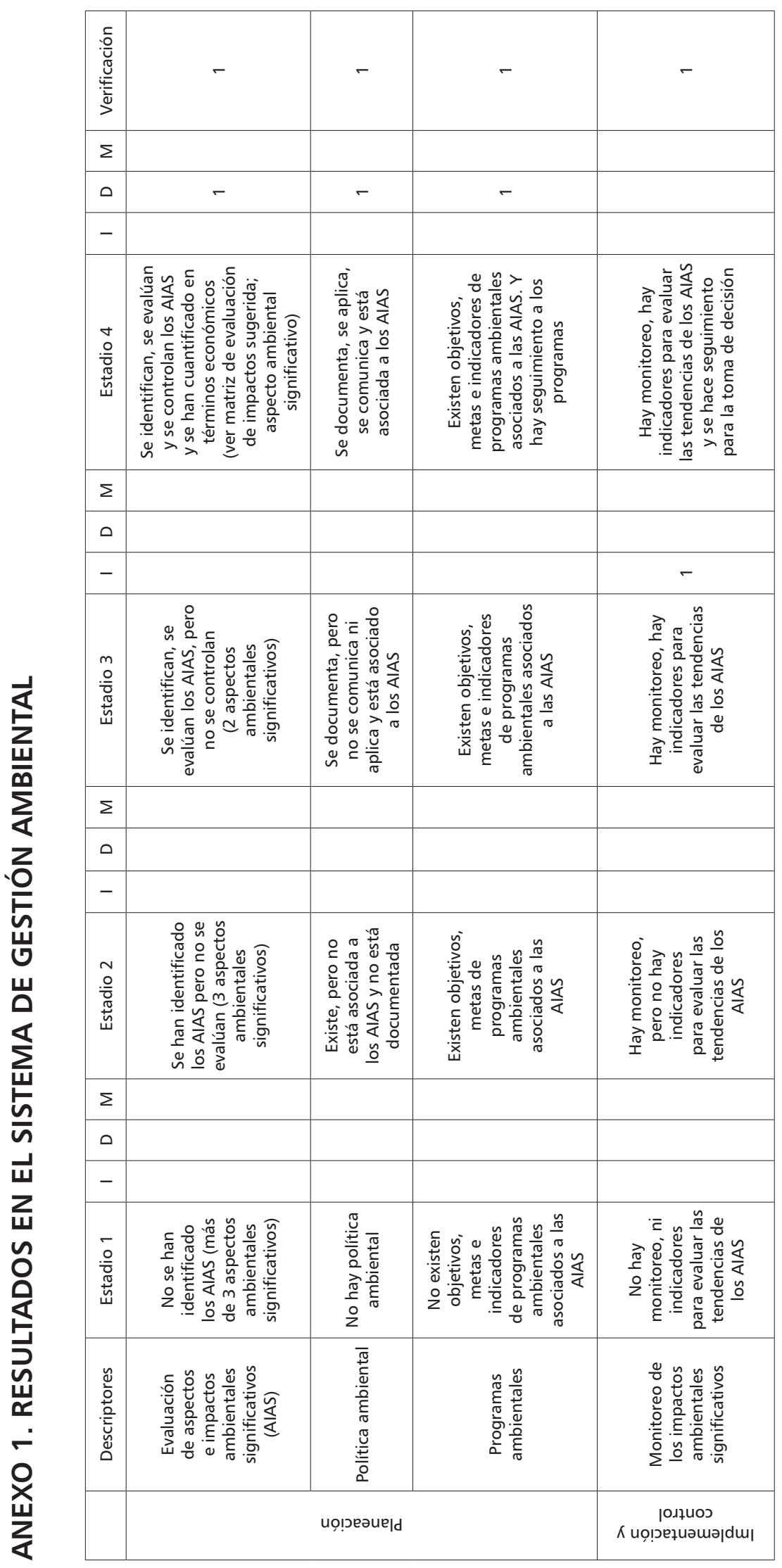




\begin{tabular}{|c|c|c|c|c|c|c|c|}
\hline 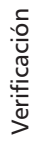 & - & - & - & & & & \\
\hline$\Sigma$ & & & & 8 & & & \\
\hline 0 & & & & $\stackrel{\substack{n \\
N}}{n}$ & & & \\
\hline- & & & & $\begin{array}{l}8 \\
0 \\
0\end{array}$ & & & \\
\hline 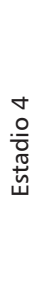 & 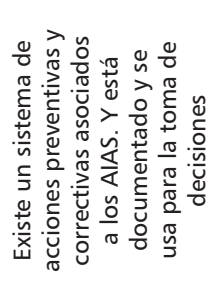 & 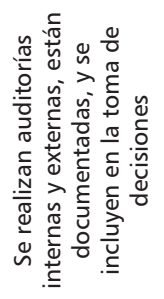 & 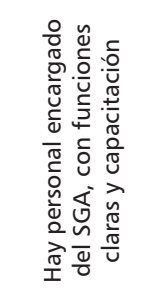 & & & & \\
\hline$\Sigma$ & & & & 8 & & & \\
\hline 0 & & - & - & $\stackrel{m}{m}$ & & & \\
\hline- & & & & $\begin{array}{l}\infty \\
\stackrel{\infty}{0} \\
0\end{array}$ & & & \\
\hline 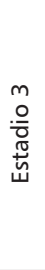 & 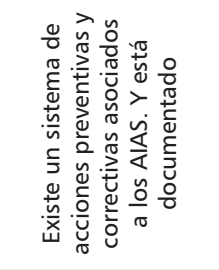 & 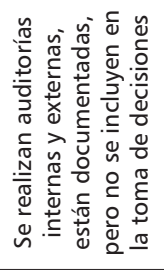 & 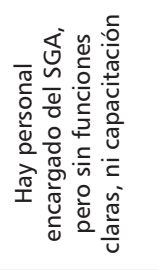 & & & & \\
\hline$\Sigma$ & & & & 8 & & & \\
\hline 0 & & & & $\begin{array}{l}8 \\
0 \\
0\end{array}$ & & & \\
\hline- & - & & & $\begin{array}{c}m \\
m \\
0\end{array}$ & & & \\
\hline 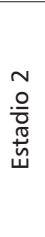 & 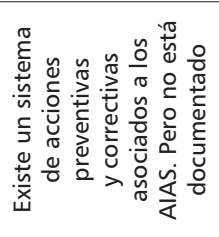 & 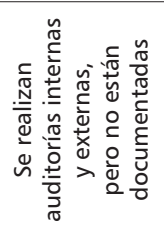 & 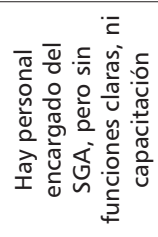 & & & & \\
\hline$\Sigma$ & & & & 8 & & & \\
\hline ○ & & & & : & & & \\
\hline- & & & & 8. & & & \\
\hline 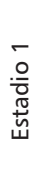 & 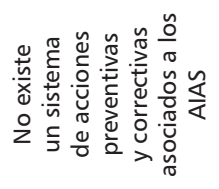 & 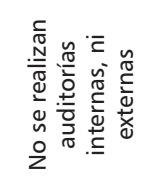 & 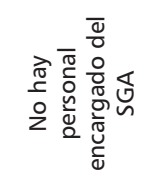 & & 只 & $\begin{array}{l}8 \\
\text { in }\end{array}$ & 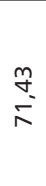 \\
\hline 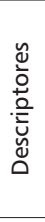 & 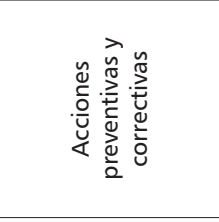 & $\begin{array}{l}\frac{n}{\frac{\pi}{2}} \\
\frac{0}{\frac{1}{7}} \\
\frac{0}{2}\end{array}$ & 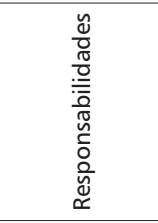 & $\begin{array}{l}\frac{\sigma}{U} \\
\frac{0}{0} \\
\frac{\pi}{0} \\
\frac{0}{0}\end{array}$ & $\begin{array}{l}\frac{0}{0} \\
\frac{0}{\bar{n}} \\
\frac{0}{\pi} \\
\frac{\pi}{0}\end{array}$ & 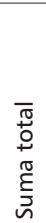 & 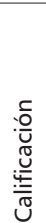 \\
\hline & \multicolumn{2}{|c|}{ 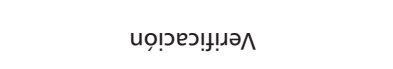 } & 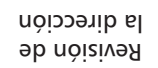 & & & & \\
\hline
\end{tabular}




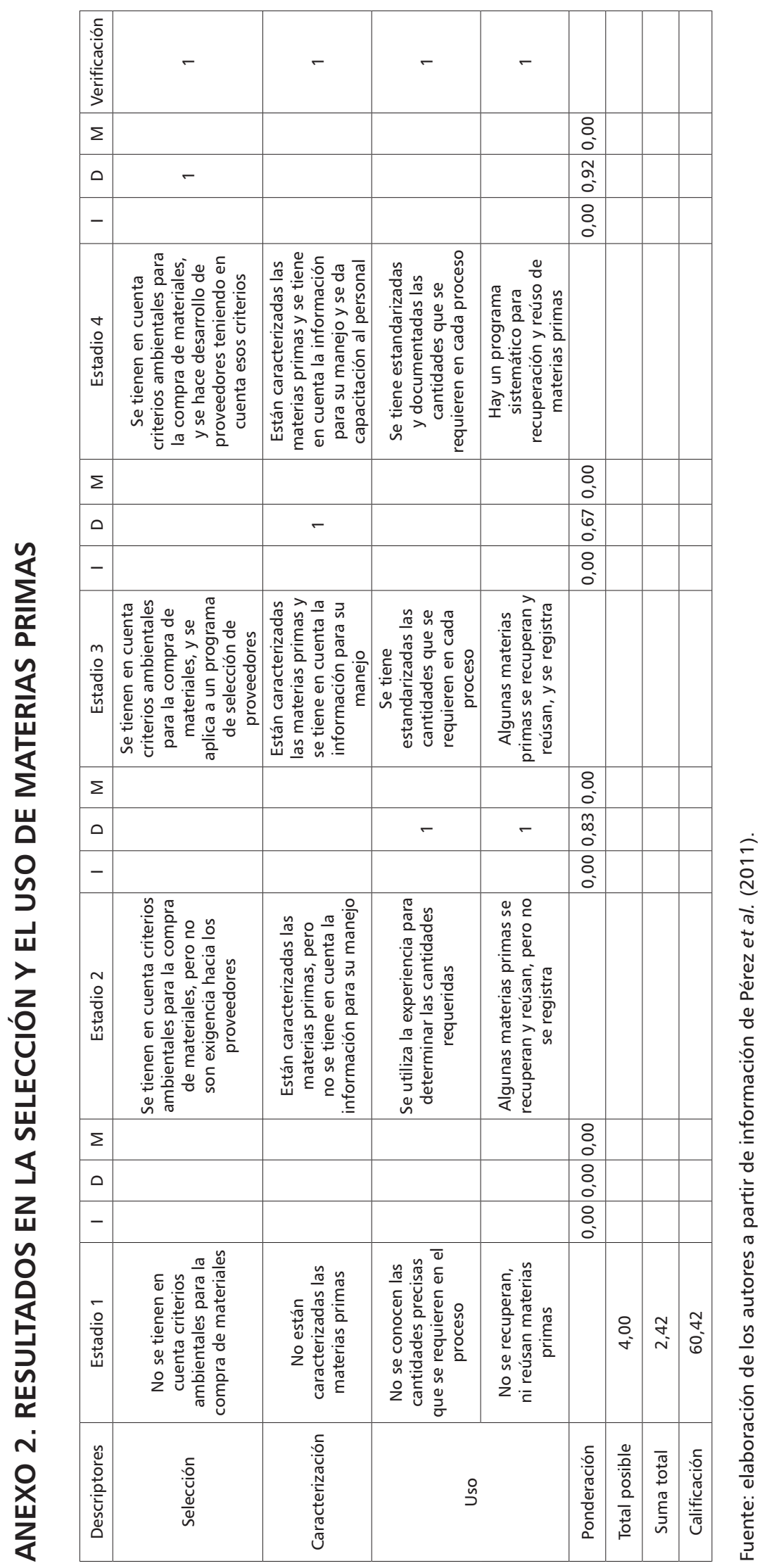




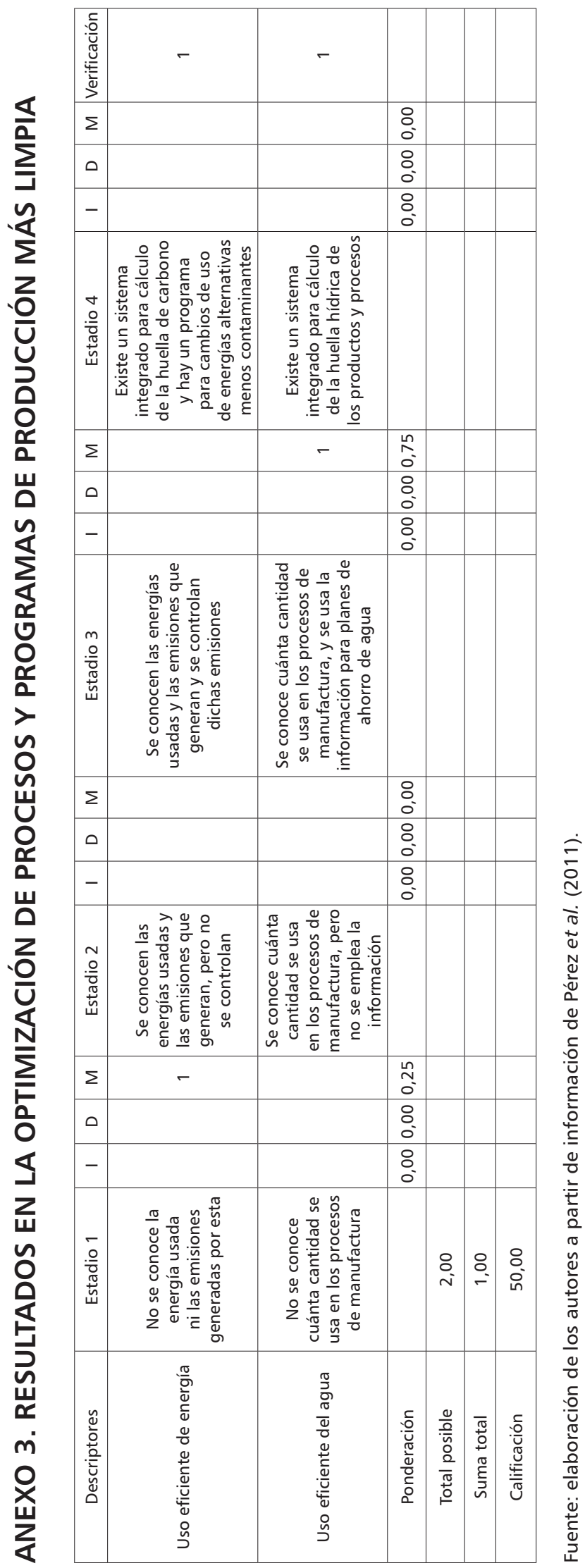




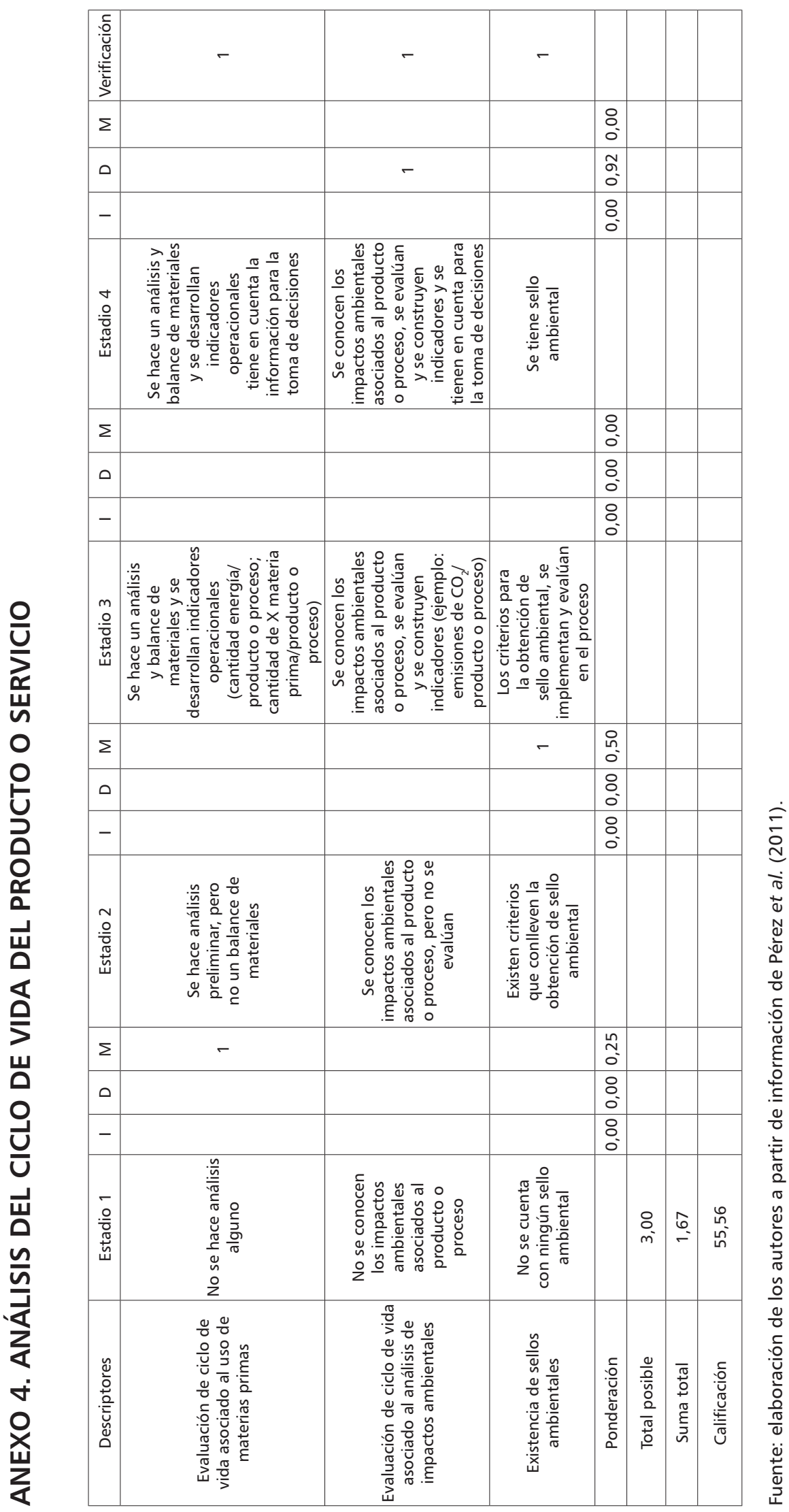




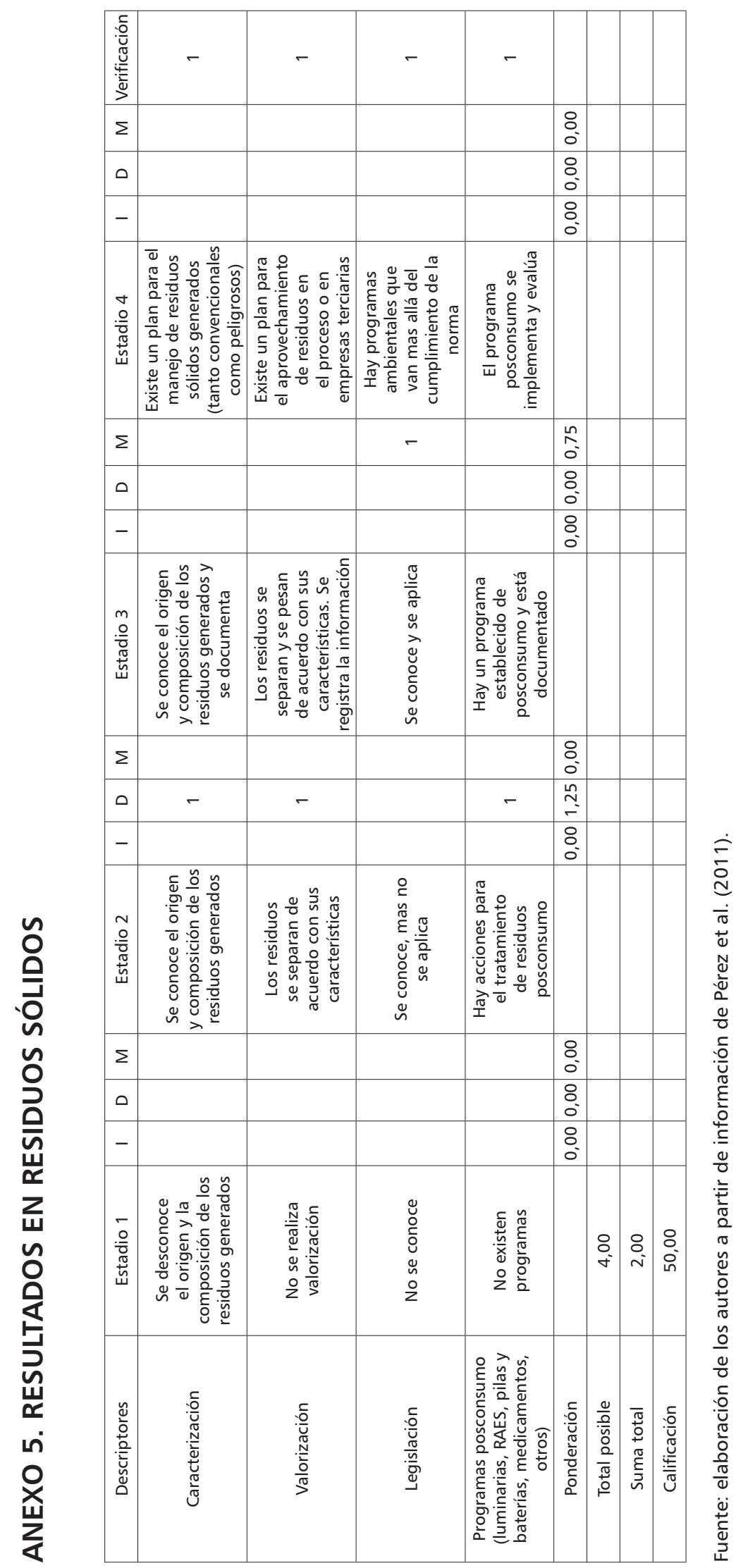



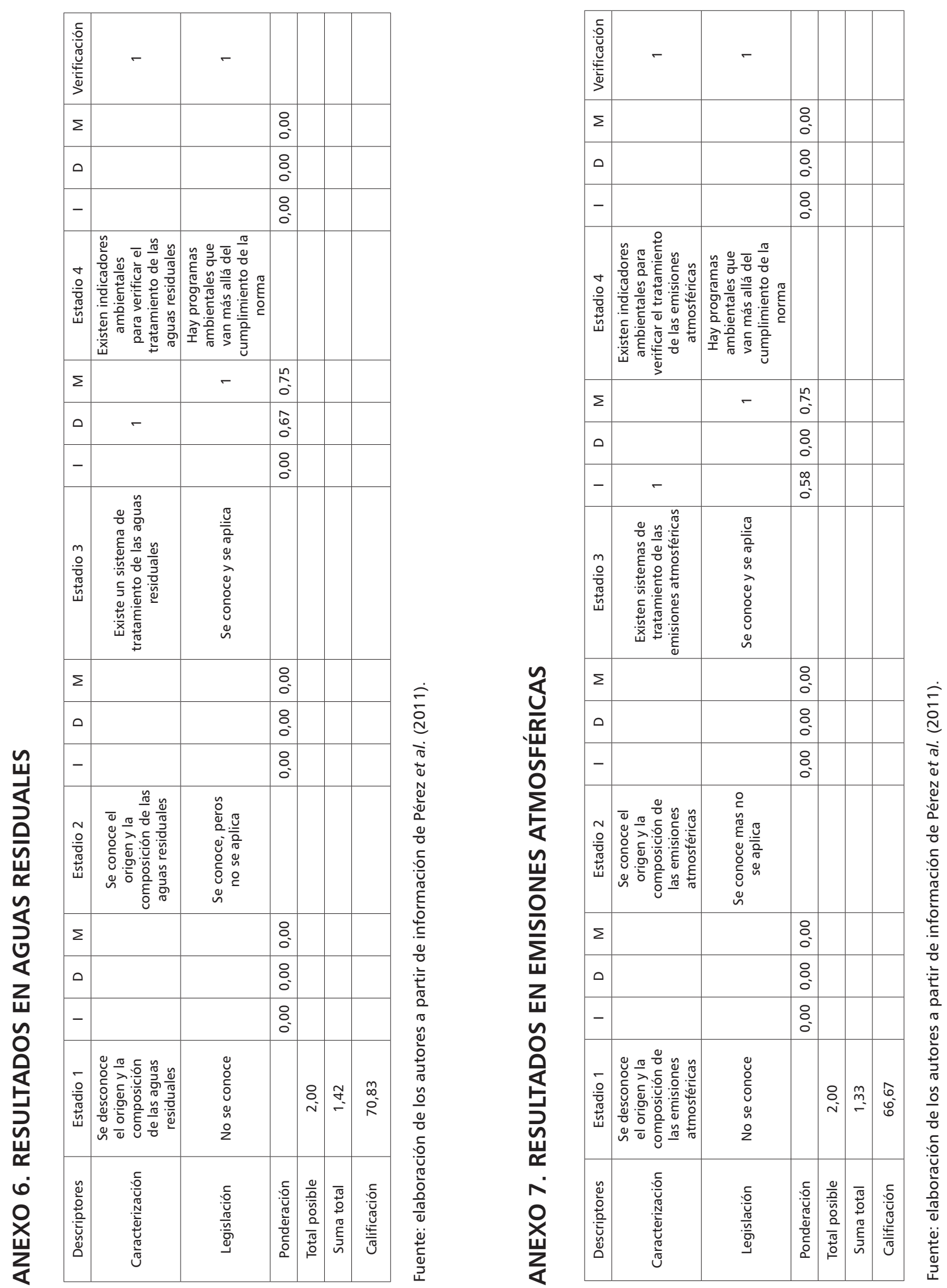

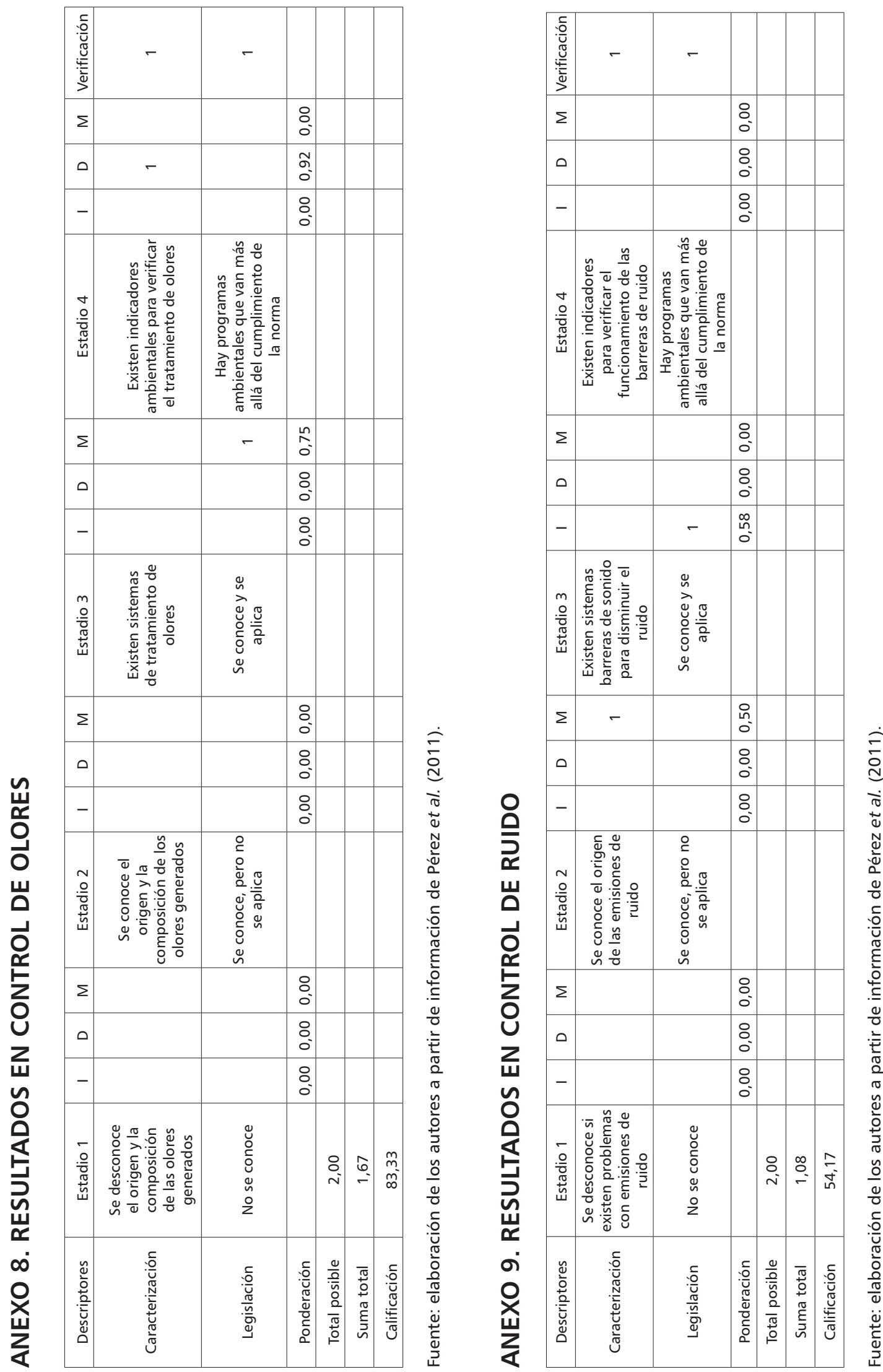


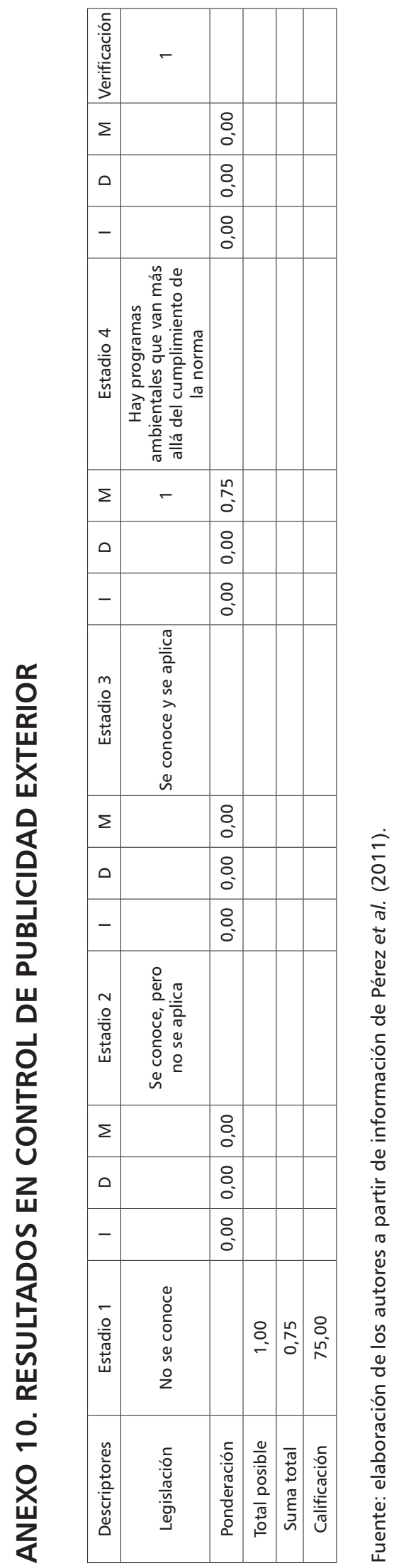




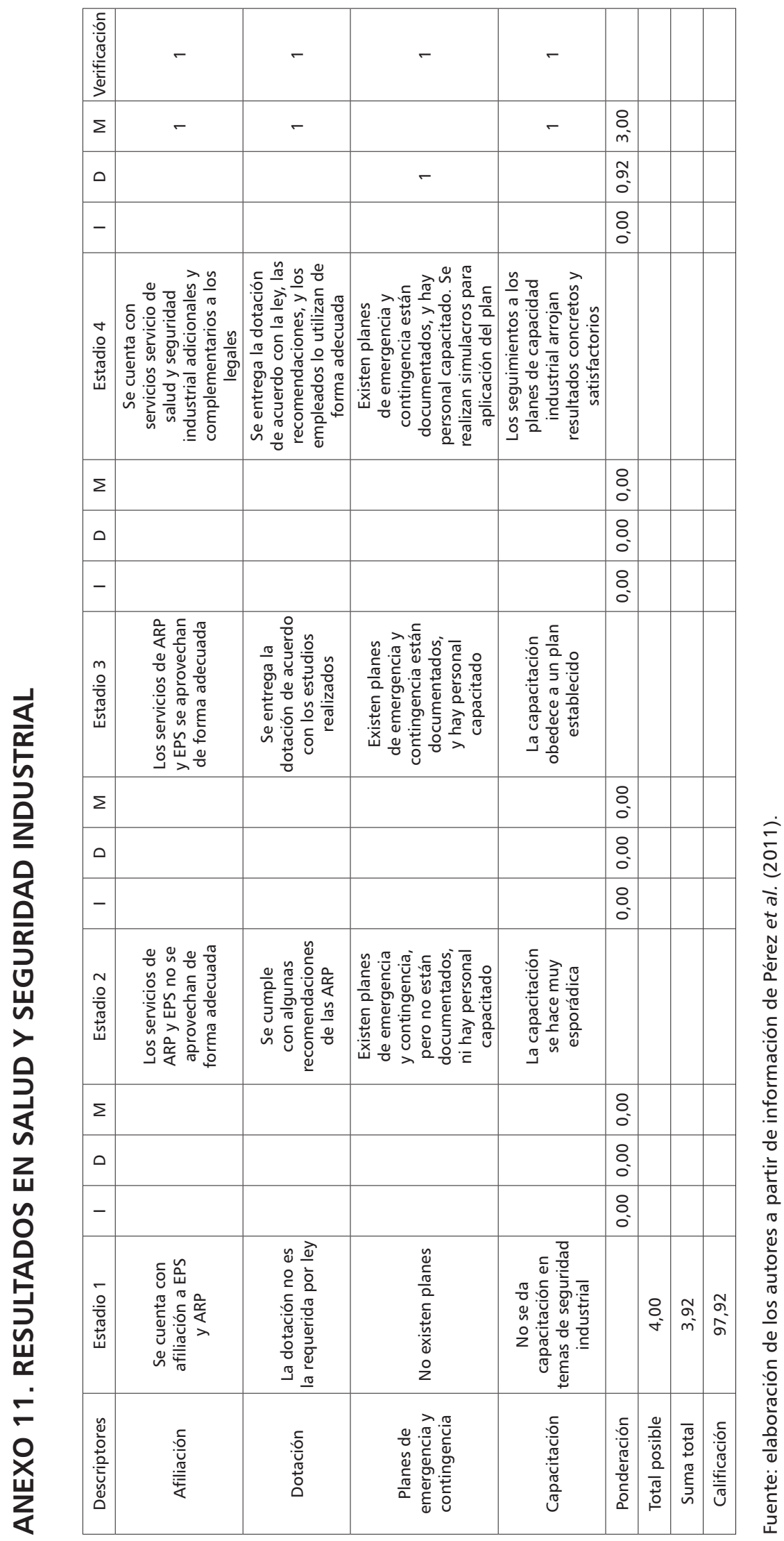



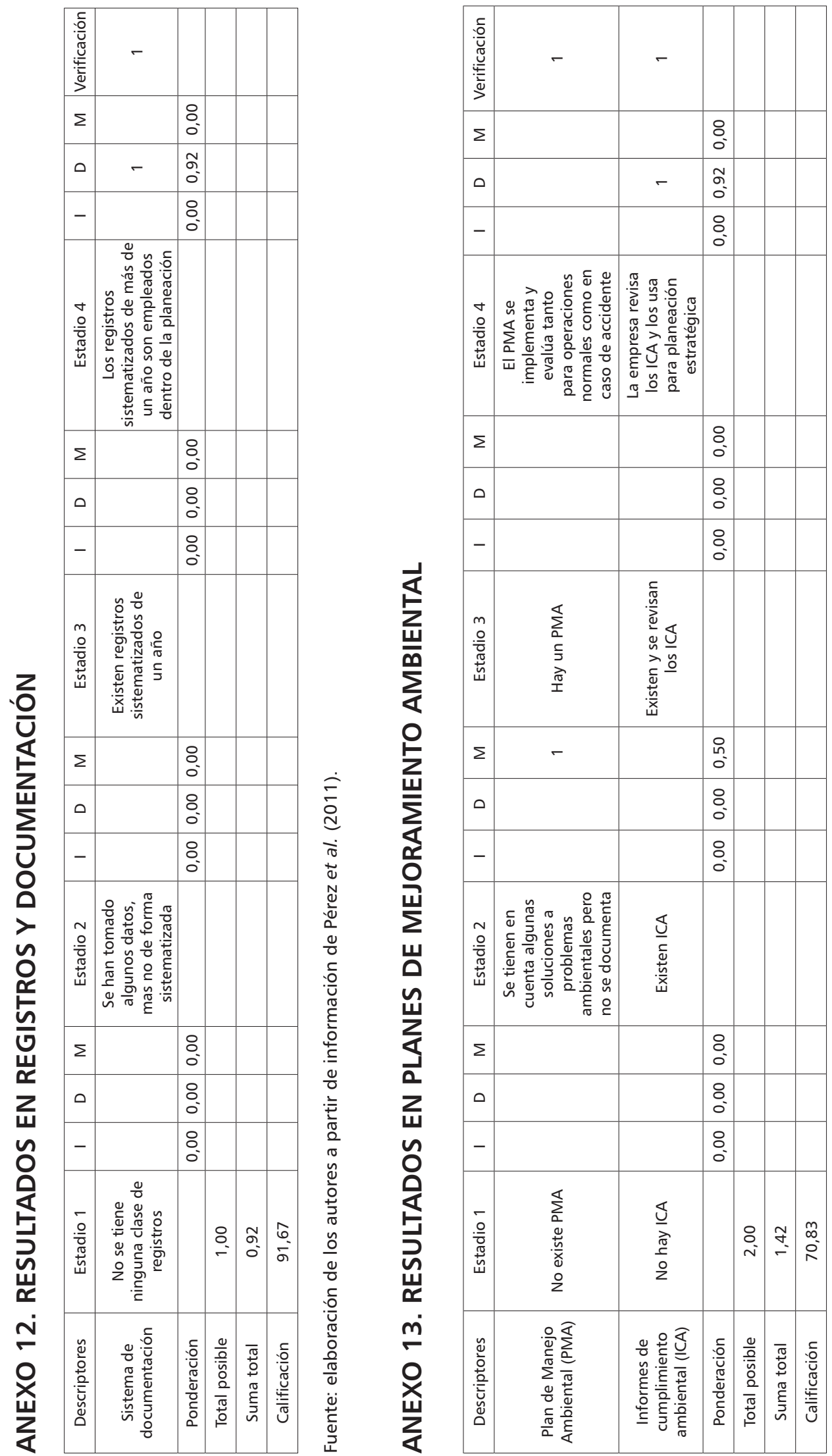

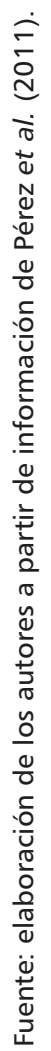




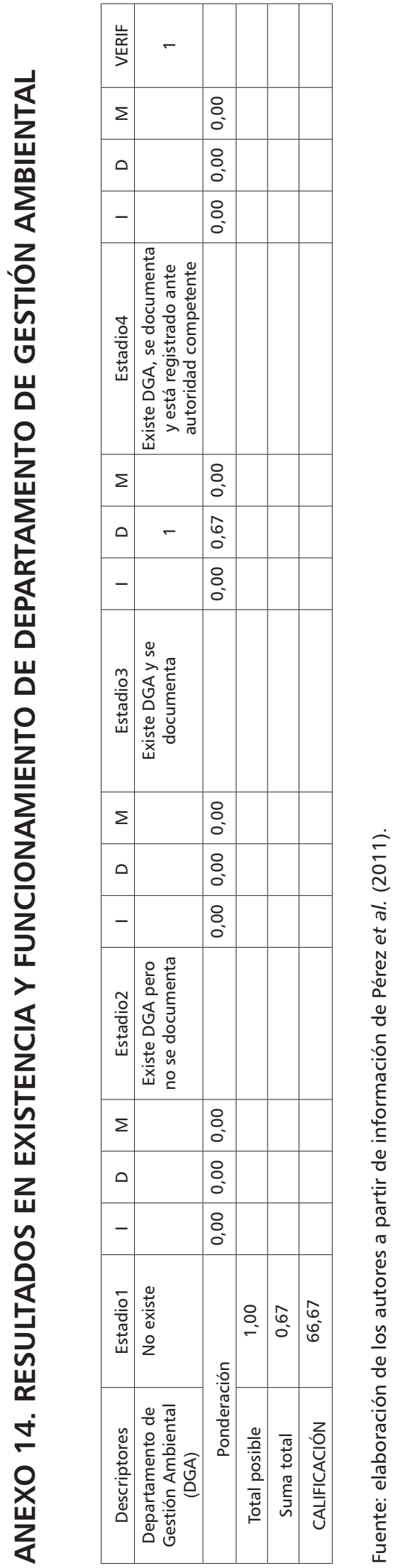




\section{ANEXO 15. ENCUESTA FOCALIZADA DIRIGIDA A LOS GERENTES O JEFES DE PRODUCCIÓN}

Ciudad y fecha:

Objetivo: Diagnosticar si en el sector industrial de la transformación de la arcilla del municipio de San José de Cúcuta y su área metropolitana se están aplicando acciones e instrumentos de gestión ambiental.

1. ¿Indique con una $x$ cuáles instrumentos de gestión ambiental de tipo preventivos que desarrolla la empresa? Marque con una $x$ su respuesta.

\begin{tabular}{|c|c|c|c|}
\hline Respuestas & $\begin{array}{c}\text { Evaluación del impacto } \\
\text { ambiental }\end{array}$ & Licencia ambiental & Plan de manejo ambiental \\
\hline Sí & & & \\
\hline No & & & \\
\hline
\end{tabular}

2. ¿Indique cuáles instrumentos de gestión ambiental de tipo correctivos que desarrolla la empresa? Marque con una $x$ su respuesta.

\begin{tabular}{|c|c|c|c|}
\hline Respuestas & Costos ambientales & Auditoría Ambiental & Contabilidad Ambiental \\
\hline Sí & & & \\
\hline No & & & \\
\hline
\end{tabular}

3. ¿Indique qué tipos de programas de mitigación ambiental aplica la empresa? Marque con una $x$ su respuesta.

\begin{tabular}{|l|l|l|l|l|l|}
\hline $\begin{array}{c}\text { Manejo de } \\
\text { combustibles }\end{array}$ & $\begin{array}{c}\text { Monitoreo de } \\
\text { emisiones }\end{array}$ & $\begin{array}{c}\text { Medidas de } \\
\text { compensación } \\
\text { ambiental }\end{array}$ & $\begin{array}{c}\text { Medidas } \\
\text { preventivas de } \\
\text { control }\end{array}$ & $\begin{array}{c}\text { Manejo de } \\
\text { residuos peligrosos }\end{array}$ & Total \\
\hline & & & & & \\
\hline
\end{tabular}

4. Considera usted que el mayor deterioro ambiental causado por las empresas del sector arcilla del departamento se realiza sobre:

$\begin{array}{ll}\text { Aire } & \square \\ \text { Agua } & \square \\ \text { Suelo } & \square \\ \text { Flora } & \square \\ \text { Fauna }\end{array}$

5. ¿La empresa realiza proyectos ambientales?

Sí

No

6. ¿En su empresa se aplica o utiliza contabilidad ambiental?

Sí

No

7. ¿Maneja su empresa indicadores de gestión ambiental?

Sí

No

8. ¿Su empresa utiliza programas de producción limpia?

Sí

No 
9. ¿Lleva la empresa registros de contaminación ambiental? Marque con una $x$ su respuesta.

\begin{tabular}{|c|c|c|c|c|c|}
\hline Respuesta & $\begin{array}{c}\text { Llevan libro de } \\
\text { contaminación }\end{array}$ & $\begin{array}{c}\text { Cumplimiento } \\
\text { de planes de } \\
\text { contingencia }\end{array}$ & $\begin{array}{c}\text { Control de sólidos } \\
\text { y líquidos }\end{array}$ & Control auditivo & Control visual \\
\hline Sí & & & & & \\
\hline No & & & & & \\
\hline
\end{tabular}

10. ¿La empresa utiliza equipos de protección para sus trabajadores? Marque con una $x$ su respuesta.

\begin{tabular}{|c|c|c|c|c|c|c|c|}
\hline Respuesta & $\begin{array}{c}\text { Uniformes } \\
(\%)\end{array}$ & $\begin{array}{c}\text { Delantal } \\
(\%)\end{array}$ & $\begin{array}{c}\text { Casco } \\
(\%)\end{array}$ & $\begin{array}{c}\text { Guantes } \\
(\%)\end{array}$ & $\begin{array}{c}\text { Protección } \\
\text { visual (\%) }\end{array}$ & $\begin{array}{c}\text { Protección } \\
\text { respiratoria }\end{array}$ & $\begin{array}{c}\text { Protección } \\
\text { auditiva }\end{array}$ \\
\hline Sí & & & & & & & \\
\hline No & & & & & & & \\
\hline
\end{tabular}

11. ¿La empresa utiliza programas de prevención ambiental? Marque con una $x$ su respuesta.

\begin{tabular}{|c|c|c|c|c|c|c|}
\hline Respuesta & $\begin{array}{c}\text { Prevención de } \\
\text { enfermedades } \\
\text { profesionales }\end{array}$ & $\begin{array}{c}\text { Prácticas de } \\
\text { manipulación } \\
\text { segura (\%) }\end{array}$ & $\begin{array}{c}\text { Superficies } \\
\text { limpias } \\
(\%)\end{array}$ & $\begin{array}{c}\text { Programas de } \\
\text { prevención } \\
\text { accidentes }\end{array}$ & $\begin{array}{c}\text { Equipos de } \\
\text { primeros } \\
\text { auxilios (\%) }\end{array}$ & $\begin{array}{c}\text { Limpieza y } \\
\text { desinfección } \\
\text { sistemática }\end{array}$ \\
\hline Sí & & & & & & \\
\hline No & & & & & & \\
\hline
\end{tabular}

12. ¿La empresa utiliza programas de RSC con los grupos de interés o stakeholders? Marque con una $x$ su respuesta.

\begin{tabular}{|c|c|c|c|c|c|c|c|}
\hline Respuesta & $\begin{array}{c}\text { Trabajadores } \\
(\%)\end{array}$ & $\begin{array}{c}\text { Accionistas } \\
(\%)\end{array}$ & $\begin{array}{c}\text { Proveedores } \\
(\%)\end{array}$ & $\begin{array}{c}\text { Clientes } \\
(\%)\end{array}$ & $\begin{array}{c}\text { Competidores } \\
(\%)\end{array}$ & $\begin{array}{c}\text { Administración } \\
\text { pública (\%) }\end{array}$ & $\begin{array}{c}\text { Comunidad } \\
(\%)\end{array}$ \\
\hline Sí & & & & & & & \\
\hline No & & & & & & & \\
\hline
\end{tabular}

13. ¿Qué porcentaje de las ventas de la empresa representan los costos ambientales? Marque con una $x$ su respuesta.

\begin{tabular}{|c|c|c|}
\hline$\#$ & Rangos en porcentajes & Respuesta \\
\hline 1 & 0 a 20 & \\
\hline 2 & 21 a 40 & \\
\hline 3 & 41 a 60 & \\
\hline 4 & 61 a 80 & \\
\hline 5 & 81 a 100 & \\
\hline
\end{tabular}

14. ¿Los costos de producción son acordes con la rentabilidad esperada? Marque con una $x$ su respuesta.

\begin{tabular}{|c|c|c|c|}
\hline Respuesta & Muy altos & Se logró reducirlos & Bajos \\
\hline Sí & & & \\
\hline No & & & \\
\hline
\end{tabular}

15. Tendencias que han marcado los costos de producción con relación a los costos en los programas para el cuidado del medioambiente. Marque con una $x$ su respuesta.

\begin{tabular}{|c|c|c|}
\hline Baja & Constante & Alta \\
\hline & & \\
\hline
\end{tabular}

